\title{
Synthetic Studies on the MARDi Cascade: Stereoselective Synthesis of Heterocyclic Seven- Membered Rings
}

Yoann Coquerel,* David Bensa, Alain Doutheau and Jean Rodriguez*

\section{Supporting Information}

\section{Experimental section}

General experimental:

$\mathrm{MeOH}$ was dried by refluxing with magnesium and then distilled under argon. $\mathrm{K}_{2} \mathrm{CO}_{3}$ was dried by prolonged storage at $140{ }^{\circ} \mathrm{C}$ in an oven. The reactions were monitored by TLC, which were performed on Merck 60F254 plates and visualized with an ethanolic solution of $p$-anisaldehyde and sulfuric acid or an ethanolic solution of molybdophosphoric acid. Flash chromatography was performed with Merck 230-400 mesh silica gel. NMR data were recorded on a Brücker Avance 200, Avance 300 or Avance 400 spectrometer in $\mathrm{CDCl}_{3}$ or $\mathrm{C}_{6} \mathrm{D}_{6}$, and chemical shifts $(\delta)$ are given in ppm relative to the residual $\mathrm{CHCl}_{3}$ signal for ${ }^{1} \mathrm{H}$ NMR (7.25 ppm) and relative to the

deutered solvent signal for ${ }^{13} \mathrm{C}$ NMR $(77.0 \mathrm{ppm})$; coupling constants $(J)$ are in Hertz, and the classical abbreviations are used to describe the signal multiplicity; peak assignment and relative configurations have been established from standard COSY and NOESY NMR data. Mass spectra were recorded on a API III Plus Sciex spectrometer.

General procedure for the preparation of diesters 3a-d:

An oven dried, two-necked, round-bottomed flask under an argon atmosphere equipped with a 
magnetic Teflon-coated stirring bar is charged at room temperature, in that order, with $1.0 \mathrm{mmol}$ of $\beta$-ketoester 1 and $25 \mathrm{~mL}$ of $\mathrm{MeOH}$. At $0{ }^{\circ} \mathrm{C}$, the required amount of $\mathrm{K}_{2} \mathrm{CO}_{3}$ (see Table 1 ) is added and the mixture is stirred for 10 minutes, and $100 \mu \mathrm{L}(1.5 \mathrm{mmol})$ of acrolein 2a are then added. The reaction mixture is slowly warmed to room temperature and stirred at that temperature for 16 hours (entries 1-4 and 8) or 30 hours (entries 5-7). Water (20 mL) is then added to the reaction mixture and methanol is removed under reduced pressure; the resulting aqueous layer is extracted three times with ethyl acetate and the combined organic layers are washed with brine, dried with anhydrous sodium sulfate, filtrated and concentrated to give the crude product. The crude product is purified by flash chromatography on silica gel eluted with increasing amount of ether or ethyl acetate in petrol ether. The acid $3 c(R=H)$ is obtained by acidification of the previously extracted aqueous layer, which is then extracted three times with ethyl acetate and the combined organic layers are dried with anhydrous sodium sulfate, filtrated and concentrated to give the clean crude product.

General procedure for the preparation of diesters $3 \mathbf{e}-\mathrm{j}$ :

An oven dried, two-necked, round-bottomed flask under an argon atmosphere equipped with a magnetic Teflon-coated stirring bar is charged at room temperature, in that order, with $1.0 \mathrm{mmol}$ of $\beta$-ketoester 1 and $25 \mathrm{~mL}$ of $\mathrm{MeOH}$. At $0{ }^{\circ} \mathrm{C}, 150 \mu \mathrm{L}$ (1.0 mmol) of DBU are added and the mixture is stirred for 10 minutes, and $1.5 \mathrm{mmol}$ of aldehyde $\mathbf{2 b}$ or $\mathbf{2} \mathbf{c}$ are then added. The reaction mixture is slowly warmed to room temperature and stirred at that temperature for 20 hours. Water (20 mL) is then added to the reaction mixture and methanol is removed under reduced pressure; the resulting aqueous layer is extracted three times with ethyl acetate and the combined organic layers are washed with brine, dried with anhydrous sodium sulfate, filtrated and concentrated to 
give the crude product. The crude product is purified by flash chromatography on silica gel eluted with increasing amount of ether or ethyl acetate in petrol ether.

General procedure for the preparation of acids 4:

An oven dried, two-necked, round-bottomed flask under an argon atmosphere equipped with a magnetic Teflon-coated stirring bar is charged at room temperature, in that order, with $1.0 \mathrm{mmol}$ of $\beta$-ketoester 1 and $25 \mathrm{~mL}$ of $\mathrm{MeOH}$. At $0{ }^{\circ} \mathrm{C}, 75 \mu \mathrm{L}(0.5 \mathrm{mmol})$ of DBU are added and the mixture is stirred for 10 minutes, and $1.5 \mathrm{mmol}$ of aldehyde $2 \mathbf{d}$ or $2 \mathbf{e}$ is then added. The reaction mixture is slowly warmed to room temperature and stirred at that temperature for 20 hours. Water (20 $\mathrm{mL}$ ) is then added to the reaction mixture and methanol is removed under reduced pressure; the resulting aqueous layer is washed twice with ethyl acetate and acidified with conc. HCl. The acidic aqueous layer is then extracted three times with ethyl acetate, and the combined organic layers are dried with anhydrous sodium sulfate, filtrated and concentrated to give the crude product. For thiepines $\mathbf{4 c , d}$ the crude product was very clean. For oxepine $\mathbf{4 a}$ and azepine $\mathbf{4 b}$, the crude product is purified by flash chromatography on silica gel eluted with increasing amount of ethyl acetate and acetic acid in petrol ether. Acetic acid could hardly be removed from the pure acids $\mathbf{4 a , b}$, and thus analytical samples were obtained as their methyl esters $\mathbf{5 a , b .}$

Preparation of diesters 5a,b:

An oven dried round-bottomed flask under an argon atmosphere equipped with a magnetic Teflon-coated stirring bar is charged at room temperature with the acid 4a,b and MeOH (0.05M). At $0{ }^{\circ} \mathrm{C}$, 2 equiv of boron trifluoride-diethyl ether complex are added and the mixture is slowly warmed to room temperature and stirred at that temperature for 13 hours. Water is then added and methanol is removed under reduced pressure; the resulting aqueous layer is extracted three times 
with ethyl acetate, and the combined organic layers are washed with brine, dried with anhydrous sodium sulfate, filtrated and concentrated to give the crude product which is purified by flash chromatography on silica gel eluted with ether in petrol ether.

\section{Characterization data:}

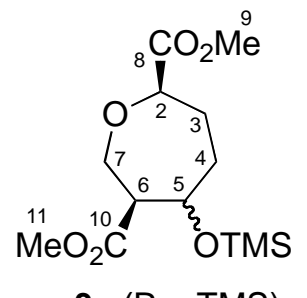

$\mathbf{3 a}(\mathrm{R}=\mathrm{TMS})$

Oxepane 3a (R = TMS, major isomer $\alpha$-OTMS): ${ }^{1} \mathrm{H}$ NMR (300 MHz, $\left.\mathrm{CDCl}_{3}\right) \delta 4.23$ (dd, $J=9.7$, $6.1 \mathrm{~Hz}, 1 \mathrm{H}, \mathrm{H} 2$ ), 4.01-4.11 (m, 1H, H5), 3.92 (dd, $J=13.1,8.2 \mathrm{~Hz}, 1 \mathrm{H}, \mathrm{H} 7$ ), 3.79 (dd, $J=13.1$, $3.1 \mathrm{~Hz}, 1 \mathrm{H}, \mathrm{H7}$ ), 3.69 (s, 3H, H9 or H11), 3.66 (s, 3H, H9 or H11), 2.69 (ddd, $J=8.2$, 8.2, 3.1 $\mathrm{Hz}, 1 \mathrm{H}, \mathrm{H} 6), 2.10-2.21$ (m, 1H, H3), 1.73-1.98 (m, 3H, H3 and H4), 0.06 (s, 9H, TMS); ${ }^{13} \mathrm{C}$ NMR (75 MHz, $\left.\mathrm{CDCl}_{3}\right) \delta 172.8(\mathrm{C}), 172.5(\mathrm{C}), 76.9(\mathrm{CH}), 73.2(\mathrm{CH}), 63.5\left(\mathrm{CH}_{2}\right), 56.0(\mathrm{CH})$, $52.1\left(\mathrm{CH}_{3}\right), 51.9\left(\mathrm{CH}_{3}\right), 32.5\left(\mathrm{CH}_{2}\right), 25.5\left(\mathrm{CH}_{2}\right), 0.1\left(\mathrm{CH}_{3}\right) ; \mathrm{MS}(\mathrm{ESI}+) \mathrm{m} / \mathrm{z} 322\left(\left[\mathrm{M}+\mathrm{NH}_{4}\right]^{+}\right.$, 100\%), $305\left([\mathrm{M}+\mathrm{H}]^{+}, 75 \%\right)$.

Minor isomer $\beta$-OTMS: ${ }^{1} \mathrm{H}$ NMR (300 MHz, $\mathrm{CDCl}_{3}$, selected resonances) $\delta 3.71$ (s, H9 or H11), 3.68 (s, H9 or H11), 0.06 (s, TMS); ${ }^{13} \mathrm{C}$ NMR (75 MHz, $\left.\mathrm{CDCl}_{3}\right) \delta 173.6$ (C), 172.3 (C), 76.5 (CH), $68.2(\mathrm{CH}), 62.2\left(\mathrm{CH}_{2}\right), 54.4(\mathrm{CH}), 52.0\left(\mathrm{CH}_{3}\right), 51.8\left(\mathrm{CH}_{3}\right), 30.6\left(\mathrm{CH}_{2}\right), 23.2\left(\mathrm{CH}_{2}\right), 0.0$ $\left(\mathrm{CH}_{3}\right)$ 


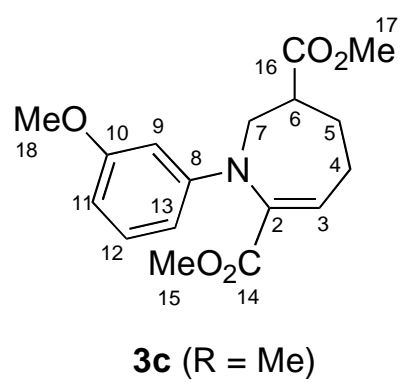

Azepine 3c (R = Me): ${ }^{1} \mathrm{H}$ NMR (300 MHz, $\left.\mathrm{CDCl}_{3}\right) \delta 7.11(\mathrm{dd}, J=7.9 \mathrm{~Hz}, 1 \mathrm{H}, \mathrm{H} 12), 6.63(\mathrm{t}, J=$ 5.6 Hz, 1H, H3), 6.33 (m, 3H, H9-H11-H13), 4.30 (dd, $J=15.1,5.1 \mathrm{~Hz}, 1 \mathrm{H}, \mathrm{H} 4$ ), 3.75 (s, 3H, H15 or H17 or H18), 3.65 (s, 3H, H15 or H17 or H18), 3.63 (s, 3H, H15 or H17 or H18), 3.63 (dd, $J=15.1,10.3 \mathrm{~Hz}, 1 \mathrm{H}, \mathrm{H} 4$ ), 3.00 (m, 1H, H6), 2.45-2.60 (m, 1H, H7), 2.21-2.34 (m, 1H, H7), 1.74-1.95 (m, 2H, H5); ${ }^{13} \mathrm{C}$ NMR (75 MHz, $\mathrm{CDCl}_{3}$ ) $\delta 174.2$ (C), 166.0 (C), 160.7 (C), 146.9 (C), $138.5(\mathrm{C}), 133.4(\mathrm{CH}), 130.0(\mathrm{CH}), 108.0(\mathrm{CH}), 104.3(\mathrm{CH}), 101.6(\mathrm{CH}), 55.1\left(\mathrm{CH}_{3}\right), 52.2\left(\mathrm{CH}_{3}\right)$, 51.8 $\left(\mathrm{CH}_{3}\right), 51.3\left(\mathrm{CH}_{2}\right), 42.1(\mathrm{CH}), 25.3\left(\mathrm{CH}_{2}\right), 25.2\left(\mathrm{CH}_{2}\right)$; MS (ESI+) m/z $320\left([\mathrm{M}+\mathrm{H}]^{+}, 100 \%\right)$.

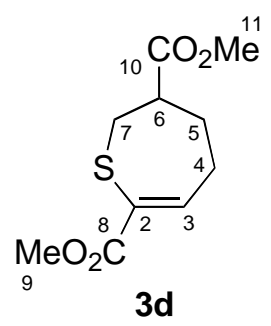

Thiepine 3d: ${ }^{1} \mathrm{H}$ NMR $\left(300 \mathrm{MHz}, \mathrm{CDCl}_{3}\right.$ ) $\delta 7.22$ (dd, $J=6.7,6.6 \mathrm{~Hz}, 1 \mathrm{H}, \mathrm{H} 3$ ), 3.75 (s, H9 or H11), 3.69 (s, H9 or H11), 3.00-3.11 (m, 2H, H7), 2.86-2.98 (m, 1H, H6), 2.59-2.72 (m, 1H, H4), 2.36-2.50 (m, 1H, H4), 2.19 (dddd, $J=14.3,9.2,4.9,1.5$ Hz, 1H, H5), 1.92 (dddd, $J$ = 14.3, 9.7, 7.9, $1.5 \mathrm{~Hz}, 1 \mathrm{H}, \mathrm{H} 5) ;{ }^{13} \mathrm{C}$ NMR (75 MHz, CDCl 3 ) $\delta 173.9$ (C), 165.7 (C), 143.5 (CH), 132.5 (C), $52.5\left(\mathrm{CH}_{3}\right), 51.9\left(\mathrm{CH}_{3}\right), 46.1(\mathrm{CH}), 34.6\left(\mathrm{CH}_{2}\right), 26.6\left(\mathrm{CH}_{2}\right), 25.5\left(\mathrm{CH}_{2}\right)$; MS (FAB+) m/z 231 ([M+H]+ $]^{+}$23\%), 136 (50\%), 73 (100\%), 39 (79\%). 


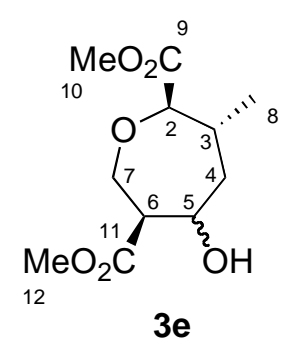

Oxepane 3e (major isomer $\alpha-\mathrm{OH}):{ }^{1} \mathrm{H}$ NMR $\left(300 \mathrm{MHz}, \mathrm{CDCl}_{3}\right) \delta$ 3.84-4.06 (m, 3H, $\mathrm{H} 5$ and $\mathrm{H7}$ ), 3.68-3.74 (m, 1H, H2), 3.72 (s, 3H, H10 or H12), 3.71 (s, 3H, H10 or H12), 2.87-2.98 (m, 1H, OH), 2.66 (ddd, $J=9.6,9.6,4.6 \mathrm{~Hz}, 1 \mathrm{H}, \mathrm{H6}$ ), 2.05-2.24 (m, 1H, H3), 1.79-1.88 (m, 2H, H4), 0.99 (d, $J=6.7 \mathrm{~Hz}, 3 \mathrm{H}, \mathrm{H8}) ;{ }^{13} \mathrm{C}$ NMR (75 MHz, $\mathrm{CDCl}_{3}$ ) $\delta 173.2$ (C), 172.6 (C), $83.3(\mathrm{CH}), 72.3$ (CH), $63.3\left(\mathrm{CH}_{2}\right), 54.5(\mathrm{CH}), 52.2\left(\mathrm{CH}_{3}\right), 51.9\left(\mathrm{CH}_{3}\right), 41.4\left(\mathrm{CH}_{2}\right), 31.6(\mathrm{CH}), 19.2\left(\mathrm{CH}_{3}\right)$; MS (FAB+) m/z 247 ([M+H] $\left.]^{+}, 15 \%\right), 229$ (15\%), 169 (19\%), 154 (44\%), 136 (51\%), 77 (58\%), 59 (100\%), 39 (82\%).

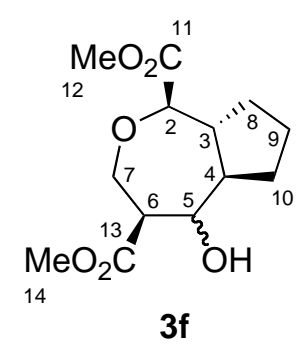

Oxepane $3 f$ (major isomer $\alpha-\mathrm{OH}):{ }^{1} \mathrm{H}$ NMR (300 MHz, $\left.\mathrm{CDCl}_{3}\right) \delta$ 3.87-3.96 (m, 3H, H5 and H7), 3.63-3.72 (m, 1H, H2), 3.70 (s, 3H, H12 or H14), 3.69 (s, 3H, H12 or H14), 2.72 (ddd, $J$ = 9.7, 8.2, $5.9 \mathrm{~Hz}, 1 \mathrm{H}, \mathrm{H6}$ ), 1.95-2.20 (m, 3H, H3-4 and H8), 1.41-1.94 (m, 5H, H8-10); ${ }^{13} \mathrm{C}$ NMR (75 MHz, $\left.\mathrm{CDCl}_{3}\right) \delta 173.2(\mathrm{C}), 173.1(\mathrm{C}), 80.8(\mathrm{CH}), 76.9(\mathrm{CH}), 62.6\left(\mathrm{CH}_{2}\right), 55.1(\mathrm{CH}), 52.1\left(\mathrm{CH}_{3}\right)$, $51.9\left(\mathrm{CH}_{3}\right), 49.3(\mathrm{CH}), 41.7(\mathrm{CH}), 31.3\left(\mathrm{CH}_{2}\right), 30.5\left(\mathrm{CH}_{2}\right), 24.1\left(\mathrm{CH}_{2}\right)$; MS (ESI+) m/z 290 $\left(\left[\mathrm{M}+\mathrm{NH}_{4}\right]^{+}, 100 \%\right), 273\left([\mathrm{M}+\mathrm{H}]^{+}, 48 \%\right)$. 
Minor isomer $\beta-\mathrm{OH}:{ }^{13} \mathrm{C}$ NMR (75 MHz, $\left.\mathrm{CDCl}_{3}\right) \delta 173.6(\mathrm{C}), 172.2(\mathrm{C}), 80.5(\mathrm{CH}), 72.5(\mathrm{CH})$, $68.0\left(\mathrm{CH}_{2}\right), 52.2\left(\mathrm{CH}_{3}\right), 51.8\left(\mathrm{CH}_{3}\right), 48.2(\mathrm{CH}), 45.0(\mathrm{CH}), 43.7(\mathrm{CH}), 30.9\left(\mathrm{CH}_{2}\right), 27.7\left(\mathrm{CH}_{2}\right)$, $25.9\left(\mathrm{CH}_{2}\right)$.
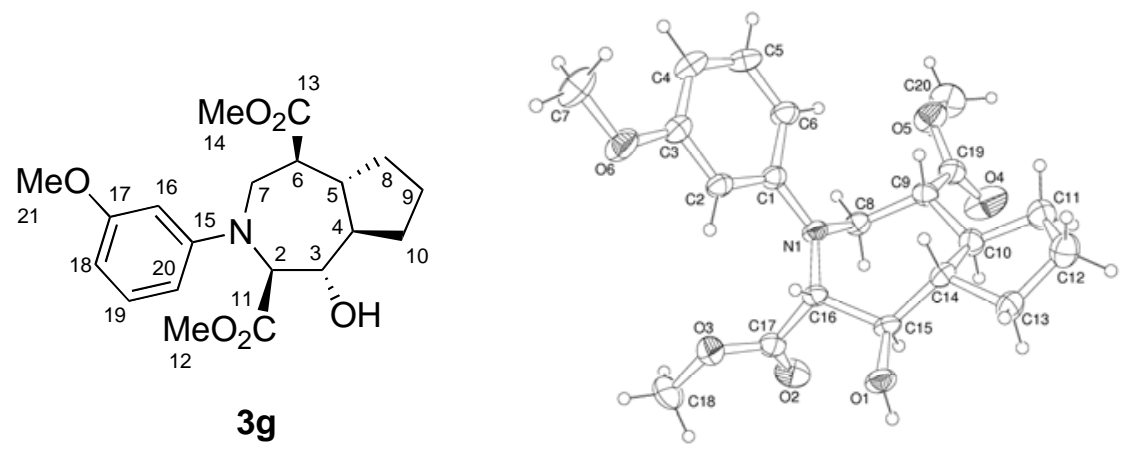

Azepane 3g: needles from $\mathrm{Et}_{2} \mathrm{O}$ :pentane at $-35{ }^{\circ} \mathrm{C}$; mp $163{ }^{\circ} \mathrm{C} ;{ }^{1} \mathrm{H}$ NMR $\left(300 \mathrm{MHz}, \mathrm{CDCl}_{3}\right) \delta$ 7.16 (dd, $J$ = 8.7, 8.7 Hz, 1H, H19), 6.32-6.40 (m, 3H, H16-H18-H20), 4.19 (dd, J = 8.2, 1.0 Hz, 1H, H2), 4.04 (ddd, $J=8.2,8.2,3.3 \mathrm{~Hz}, 1 \mathrm{H}, \mathrm{H} 3$ ), 3.79 (s, 3H, H12 or H13 or H21), 3.75 (s, 3H, H12 or H13 or H21), 3.73 (s, 3H, H12 or H13 or H21), 3.70 (dd, $J=15.6,3.7 \mathrm{~Hz}, 1 \mathrm{H}, \mathrm{H} 7), 3.28$ (dd, $J=15.6,10.8 \mathrm{~Hz}, 1 \mathrm{H}, \mathrm{H7}$ ), 2.79 (d, $J=3.3 \mathrm{~Hz}, 1 \mathrm{H}, \mathrm{OH}$ ), 2.51-2.60 (m, 1H, H6), 1.77-2.07 (m, 4H, H4-H5-H8-H10), 1.47-1.75 (m, 2H, H8-H10), 1.17-1.32 (m, 2H, H9); ${ }^{13} \mathrm{C}$ NMR (75 MHz, $\left.\mathrm{CDCl}_{3}\right) \delta 174.2(\mathrm{C}), 173.9(\mathrm{C}), 160.9$ (C), $149.0(\mathrm{C}), 130.2(\mathrm{CH}), 104.5(\mathrm{CH}), 102.5(\mathrm{CH})$, 98.2 (CH), $79.3(\mathrm{CH}), 66.4(\mathrm{CH}), 54.8\left(\mathrm{CH}_{3}\right), 52.2\left(\mathrm{CH}_{3}\right), 51.6\left(\mathrm{CH}_{3}\right), 49.2\left(\mathrm{CH}_{2}\right), 48.5(\mathrm{CH})$, $48.5(\mathrm{CH}) 48.3(\mathrm{CH}), 31.6\left(\mathrm{CH}_{2}\right), 29.4\left(\mathrm{CH}_{2}\right), 21.6\left(\mathrm{CH}_{2}\right)$; MS (ESI+) m/z $378\left([\mathrm{M}+\mathrm{H}]^{+}, 100 \%\right)$. The ORTEP view of $\mathbf{3 g}$ shows the non-depicted enantiomer (ellipsoids are at 30\% probability). The CIF for $\mathbf{3 g}$ is available free of charge via the Internet at http://pubs.acs.org. 


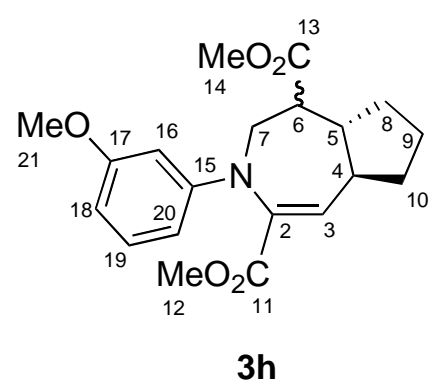

Azepine 3h (major isomer $\beta-\mathrm{CO}_{2} \mathrm{Me}$ ): ${ }^{1} \mathrm{H}$ NMR $\left(300 \mathrm{MHz}, \mathrm{CDCl}_{3}\right) \delta 7.11$ (dd, $J=8.1,8.1 \mathrm{~Hz}$, 1H, H19), 6.53 (d, $J=2.3$ Hz, 1H, H3), 6.17-6.42 (m, 3H, H16-H18-H20), 4.20-4.34 (broad dd, $J$ undetermined, 1H, H7), 3.69-3.82 (masked dd, J undetermined, 1H, H7), 3.75 (s, 3H, H12 or H13 or H21), 3.64 (s, 3H, H12 or H13 or H21), 3.63 (s, 3H, H12 or H13 or H21), 3.22-3.35 (m, 1H, H6), 3.06-3.19 (m, 1H, H4), 1.35-2.12 (m, 7H, H5 and H8-10); ${ }^{13} \mathrm{C}$ NMR (75 MHz, $\left.\mathrm{CDCl}_{3}\right) \delta$ 173.5 (C), 166.2 (C), 160.6 (C), 147.1 (C), 136.7 (C), 135.4 (broad CH), 129.9 (CH), 108.6 (CH), 104.6 (CH), $102.3(\mathrm{CH}), 55.1\left(\mathrm{CH}_{3}\right), 52.2\left(\mathrm{CH}_{2}\right), 52.2\left(\mathrm{CH}_{3}\right), 51.3\left(\mathrm{CH}_{3}\right), 43.6(\mathrm{CH}), 42.0(\mathrm{CH})$, $40.6(\mathrm{CH}), 32.0\left(\mathrm{CH}_{2}\right), 29.2\left(\mathrm{CH}_{2}\right), 22.8\left(\mathrm{CH}_{2}\right)$; MS (ESI+) m/z $360\left([\mathrm{M}+\mathrm{H}]^{+}, 100 \%\right)$.

Minor isomer $\alpha-\mathrm{CO}_{2} \mathrm{Me}:{ }^{13} \mathrm{C}$ NMR (75 MHz, $\mathrm{CDCl}_{3}$ ) $\delta 173.9$ (C), 165.7 (C), 160.8 (C), 146.4 (C), 138.1 (C), 135.8 (broad CH), $130.1(\mathrm{CH}), 106.6(\mathrm{CH}), 103.4(\mathrm{CH}), 100.3(\mathrm{CH}), 55.1\left(\mathrm{CH}_{3}\right)$, $52.1\left(\mathrm{CH}_{3}\right), 51.7\left(\mathrm{CH}_{3}\right), 50.2\left(\mathrm{CH}_{2}\right), 49.9(\mathrm{CH}), 44.1(\mathrm{CH}), 43.6(\mathrm{CH}), 32.3\left(\mathrm{CH}_{2}\right), 31.6\left(\mathrm{CH}_{2}\right)$, $22.6\left(\mathrm{CH}_{2}\right)$.

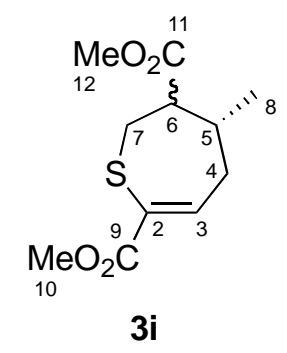

Thiepine 3i (major isomer $\beta-\mathrm{CO}_{2} \mathrm{Me}$ ): ${ }^{1} \mathrm{H} \mathrm{NMR}\left(300 \mathrm{MHz}, \mathrm{CDCl}_{3}\right) \delta 7.21$ (dd, $J=7.4,6.3 \mathrm{~Hz}$, 
1H, H3), 3.73 (s, 3H, H10 or H12), 3.67 (s, 3H, H10 or H12), 3.02 (dd, J = 14.6, 3.6 Hz, 1H, H7), 2.83 (dd, $J=14.6,7.0 \mathrm{~Hz}, 1 \mathrm{H}, \mathrm{H7}$ ), 2.55 (ddd, $J=9.1,7.0,3.5 \mathrm{~Hz}, 1 \mathrm{H}, \mathrm{H6}$ ), 2.41-2.48 (m, 2H, H4), 2.19-2.32 (m, 1H, H5), 0.97 (d, $J=6.7 \mathrm{~Hz}, 3 \mathrm{H}, \mathrm{H} 8) ;{ }^{13} \mathrm{C}$ NMR $\left(75 \mathrm{MHz}, \mathrm{CDCl}_{3}\right) \delta 174.0$ (C), $165.6(\mathrm{C}), 143.4(\mathrm{CH}), 133.0(\mathrm{C}), 53.6(\mathrm{CH}), 52.5\left(\mathrm{CH}_{3}\right), 51.8\left(\mathrm{CH}_{3}\right), 35.3\left(\mathrm{CH}_{2}\right), 34.2$ ( $\left.\mathrm{CH}_{2}\right), 30.8(\mathrm{CH}), 21.2\left(\mathrm{CH}_{3}\right) ; \mathrm{MS}(\mathrm{FAB}+)$ m/z 245 ([M+H]+, 8\%), 207 (11\%), 185 (12\%), 147 (26\%), 73 (100\%).

Minor isomer $\alpha-\mathrm{CO}_{2} \mathrm{Me}:{ }^{1} \mathrm{H}$ NMR (300 MHz, $\mathrm{CDCl}_{3}$, selected resonances) $\delta 3.73$ (s, 3H, H10 or H12), 3.64 (s, 3H, H10 or H12), 0.84 (d, $J=7.1 \mathrm{~Hz}, 3 \mathrm{H}, \mathrm{H8}) ;{ }^{13} \mathrm{C}$ NMR $\left(75 \mathrm{MHz}, \mathrm{CDCl}_{3}\right) \delta$ $173.7(\mathrm{C}), 165.7(\mathrm{C}), 142.7(\mathrm{CH}), 132.0(\mathrm{C})$, masked $(\mathrm{CH}), 51.6\left(\mathrm{CH}_{3}\right), 51.0\left(\mathrm{CH}_{3}\right), 33.7\left(\mathrm{CH}_{2}\right)$, $31.0\left(\mathrm{CH}_{2}\right), 30.3(\mathrm{CH}), 16.7\left(\mathrm{CH}_{3}\right)$.

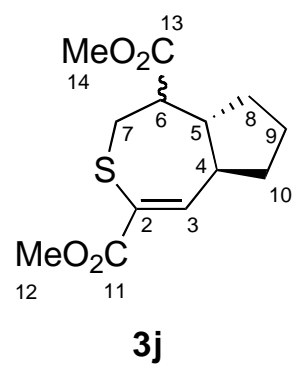

Thiepine 3j (major isomer $\beta-\mathrm{CO}_{2} \mathrm{Me}$ ): ${ }^{1} \mathrm{H}$ NMR (300 MHz, $\left.\mathrm{CDCl}_{3}\right) \delta 7.10(\mathrm{~d}, J=3.2 \mathrm{~Hz}, 1 \mathrm{H}$, H3), 3.73 (s, 3H, H12 or H14), 3.66 (s, 3H, H12 or H14), 3.28-3.39 (m, 1H, H4), 3.32 (dd, $J=$ 14.3, 10.2 Hz, 1H, H7), 3.10-3.19 (m, 1H, H6), 2.85 (dd, $J=14.2,4.5$ Hz, 1H, H7), 2.45-2.64 (m, 1H, H5), 1.99-2.13 (m, 1H, H10), 1.31-1.93 (m, 5H, H8-10); ${ }^{13} \mathrm{C}$ NMR (75 MHz, CDCl $) \delta 173.5$ (C), $165.8(\mathrm{C}), 147.2(\mathrm{CH}), 129.8(\mathrm{C}), 52.5\left(\mathrm{CH}_{3}\right), 51.5\left(\mathrm{CH}_{3}\right), 47.3(\mathrm{CH}), 42.3(\mathrm{CH}), 40.1(\mathrm{CH})$, $35.5\left(\mathrm{CH}_{2}\right), 33.9\left(\mathrm{CH}_{2}\right), 30.2\left(\mathrm{CH}_{2}\right), 24.3\left(\mathrm{CH}_{2}\right)$; MS (ESI+) m/z $271\left([\mathrm{M}+\mathrm{H}]^{+}, 100 \%\right)$.

Minor isomer $\alpha-\mathrm{CO}_{2} \mathrm{Me}:{ }^{1} \mathrm{H}$ NMR (300 MHz, $\left.\mathrm{CDCl}_{3}\right) \delta 7.30$ (d, $\left.J=5.0 \mathrm{~Hz}, 1 \mathrm{H}, \mathrm{H} 3\right), 3.74$ (s, 
3H, H12 or H14), 3.65 (s, 3H, H12 or H14), 2.89-3.01 (m, 1H, H7), 2.65-2.79 (m, 1H, H4), 2.562.63 (m, 1H, H7), 2.01-2.16 (m, 1H, H10), 1.50-2.00 (m, 6H, H5-6 and H8-10), 1.25-1.41 (m, 1H, H9); ${ }^{13} \mathrm{C}$ NMR (75 MHz, $\mathrm{CDCl}_{3}$ ) $\delta 174.1$ (C), 165.9 (C), 151.9 (CH), 131.5 (C), 53.9 (CH), $52.5\left(\mathrm{CH}_{3}\right), 51.8\left(\mathrm{CH}_{3}\right), 45.6(\mathrm{CH}), 43.1(\mathrm{CH}), 35.0\left(\mathrm{CH}_{2}\right), 33.7\left(\mathrm{CH}_{2}\right), 32.4\left(\mathrm{CH}_{2}\right), 23.5\left(\mathrm{CH}_{2}\right)$; MS (ESI+) $m / z 271\left([\mathrm{M}+\mathrm{H}]^{+}, 100 \%\right)$.

A very minor unknown product could not be removed from both diastereomers of $3 \mathbf{j}$ (see spectra).

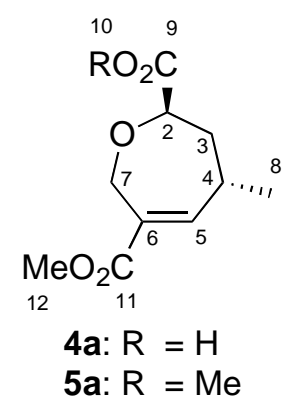

Oxepines 4a: ${ }^{1} \mathrm{H}$ NMR (300 MHz, $\mathrm{CDCl}_{3}$ ) $\delta 7.37$ (broad s, $\left.1 \mathrm{H}, \mathrm{H} 10\right), 6.84$ (d, $J=4.2 \mathrm{~Hz}, 1 \mathrm{H}$, H5), 4.73 (d, $J=16.6 \mathrm{~Hz}, 1 \mathrm{H}, \mathrm{H7}$ ), 4.44 (d, $J=16.6 \mathrm{~Hz}, 1 \mathrm{H}, \mathrm{H7}$ ), 4.41 (dd, $J=6.3,6.3 \mathrm{~Hz}, 1 \mathrm{H}$, H2), 3.67 (s, 3H, H12), 2.69-2.86 (m, 1H, H4), 2.13-2.21 (m, 2H, H3), 1.14 (d, J = 7.2 Hz, 3H, H8); ${ }^{13} \mathrm{C}$ NMR (75 MHz, CDCl $) \delta 175.3$ (C), 166.4 (C), 148.8 (CH), 130.7 (C), 75.1 (CH), 65.0 $\left(\mathrm{CH}_{2}\right), 51.8\left(\mathrm{CH}_{3}\right), 36.2\left(\mathrm{CH}_{2}\right), 30.0(\mathrm{CH}), 20.1\left(\mathrm{CH}_{3}\right)$.

Oxepine 5a: ${ }^{1} \mathrm{H}$ NMR (300 MHz, $\mathrm{CDCl}_{3}$ ) $\delta 6.88$ (ddd, $J=4.4,2.2,1.8 \mathrm{~Hz}, 1 \mathrm{H}, \mathrm{H} 5$ ), 4.75 (ddd, $J$ = 16.6, 1.8, $1.8 \mathrm{~Hz}, 1 \mathrm{H}, \mathrm{H7}$ ), 4.44 (ddd, $J=16.6,2.3,1.5 \mathrm{~Hz}, 1 \mathrm{H}, \mathrm{H7}$ ), 4.41 (dd, $J=6.7,6.7 \mathrm{~Hz}$, 1H, H2), 3.75 (s, 3H, H10 or H12), 3.70 (s, 3H, H10 or H12), 2.75-2.92 (m, 1H, H4), 2.18 (ddd, $J$ = 14.6, 6.9, $3.3 \mathrm{~Hz}, 1 \mathrm{H}, \mathrm{H3}$ ), 2.10 (ddd, $J=14.6,8.2,6.4 \mathrm{~Hz}, 1 \mathrm{H}, \mathrm{H3}), 1.17$ (d, $J=7.4 \mathrm{~Hz}, 3 \mathrm{H}$, H8); ${ }^{13} \mathrm{C}$ NMR (75 MHz, $\mathrm{CDCl}_{3}$ ) $\delta 172.4(\mathrm{C}), 166.2$ (C), $148.4(\mathrm{CH}), 131.0$ (C), $75.1(\mathrm{CH}), 64.9$ 
$\left(\mathrm{CH}_{2}\right), 52.0\left(\mathrm{CH}_{3}\right), 51.6\left(\mathrm{CH}_{3}\right), 36.2\left(\mathrm{CH}_{2}\right), 30.0(\mathrm{CH}), 19.7\left(\mathrm{CH}_{3}\right) ; \mathrm{MS}(\mathrm{ESI}+) \mathrm{m} / \mathrm{z} 246$ $\left(\left[\mathrm{M}+\mathrm{NH}_{4}\right]^{+}, 100 \%\right), 229\left([\mathrm{M}+\mathrm{H}]^{+}, 60 \%\right)$.

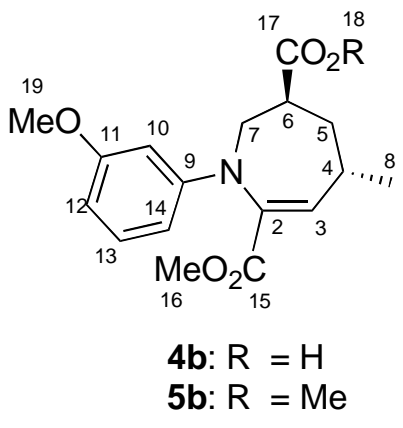

Azepine 4b: ${ }^{1} \mathrm{H}$ NMR (300 MHz, $\mathrm{CDCl}_{3}$ ) $\delta 10.87$ (broad s, $1 \mathrm{H}, \mathrm{H} 18$ ), 7.15 (dd, $J=8.2,8.2 \mathrm{~Hz}$, 1H, H13), 6.30-6.46 (m, 4H, H3-H10-H12-H14), 4.33 (dd, $J=14.9,5.9$ Hz, 1H, H7), 3.78 (dd, $J$ = 14.9, 3.6 Hz, 1H, H7), 3.78 (s, 3H, H16 or H19), 3.66 (s, 3H, H16 or H19), 3.10-3.21 (m, 1H, H6), 2.74-2.91 (m, 1H, H4), 1.86 (broad d, $J=14.6 \mathrm{~Hz}, \mathrm{H}$ ), 1.72 (ddd, $J=14.6,10.8,6.1 \mathrm{~Hz}$, H5), 1.15 (d, $J=7.2 \mathrm{~Hz}, \mathrm{H8}) ;{ }^{13} \mathrm{C}$ NMR (75 MHz, $\left.\mathrm{CDCl}_{3}\right) \delta 180.1$ (C), 166.3 (C), 160.6 (C), $147.0(\mathrm{C}), 137.6(\mathrm{CH}), 136.6(\mathrm{C}), 130.0(\mathrm{CH}), 108.5(\mathrm{CH}), 104.7(\mathrm{CH}), 102.0(\mathrm{CH}), 55.1\left(\mathrm{CH}_{3}\right)$, 52.2 $\left(\mathrm{CH}_{3}\right), 51.0\left(\mathrm{CH}_{2}\right), 40.3(\mathrm{CH}), 32.5\left(\mathrm{CH}_{2}\right), 30.5(\mathrm{CH}), 22.2\left(\mathrm{CH}_{3}\right)$.

Azepine 5b: ${ }^{1} \mathrm{H}$ NMR (300 MHz, $\left.\mathrm{CDCl}_{3}\right) \delta 7.11$ (dd, $\left.J=8.2,8.2 \mathrm{~Hz}, 1 \mathrm{H}, \mathrm{H} 13\right), 6.26-6.41(\mathrm{~m}$, 4H, H3-H10-H12-H14), 4.23 (dd, $J=15.1,5.9$ Hz, 1H, H7), 3.75 (dd, $J=15.1,7.2$ Hz, 1H, H7), 3.74 (s, 3H, H16 or H18 or H19), 3.65 (s, 3H, H16 or H18 or H19), 3.62 (s, 3H, H16 or H18 or H19), 3.02-3.14 (m, 1H, H6), 2.71-2.86 (m, 1H, H4), 1.86 (ddd, $J$ = 14.3, 3.4, 3.2 Hz, H5), 1.72 (ddd, $J=14.3,10.8,6.1 \mathrm{~Hz}, \mathrm{H} 5), 1.11$ (d, $J=7.2 \mathrm{~Hz}, \mathrm{H} 8) ;{ }^{13} \mathrm{C}$ NMR $\left(75 \mathrm{MHz}, \mathrm{CDCl}_{3}\right) \delta 174.3$ (C), 166.1 (C), 160.6 (C), 147.1 (C), 137.7 (CH), 136.6 (C), 129.9 (CH), 108.4 (CH), 104.5 (CH), 102.0 (CH), $55.1\left(\mathrm{CH}_{3}\right), 52.1\left(\mathrm{CH}_{3}\right), 51.7\left(\mathrm{CH}_{3}\right), 51.1\left(\mathrm{CH}_{2}\right), 40.3(\mathrm{CH}), 32.8\left(\mathrm{CH}_{2}\right), 30.4(\mathrm{CH})$, $22.1\left(\mathrm{CH}_{3}\right) ; \mathrm{MS}(\mathrm{ESI}+) \mathrm{m} / \mathrm{z} 334\left([\mathrm{M}+\mathrm{H}]^{+}, 100 \%\right)$. 


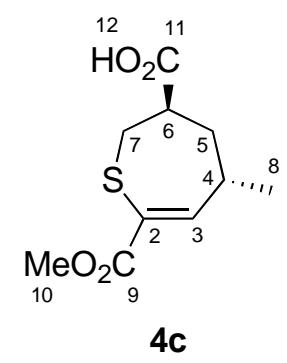

Thiepine 4c: ${ }^{1} \mathrm{H}$ NMR (300 MHz, $\left.\mathrm{CDCl}_{3}\right) \delta 8.35$ (broad s, $1 \mathrm{H}, \mathrm{H12}$ ), 6.96 (d, $J=4.9 \mathrm{~Hz}, 1 \mathrm{H}$, H3), 3.76 (s, 3H, H10), 3.28 (dd, $J=14.1,7.7$ Hz, 1H, H7), 3.03-3.13 (m, 1H, H6), 2.89-3.01 (m, 1H, H4), 2.89 (dd, $J=14.1,4.1 \mathrm{~Hz}, 1 \mathrm{H}, \mathrm{H} 7$ ), 2.07 (ddd, $J=14.3,4.8,1.8 \mathrm{~Hz}, 1 \mathrm{H}, \mathrm{H} 5$ ), 1.99 (ddd, $J=14.3,9.7,5.1 \mathrm{~Hz}, 1 \mathrm{H}, \mathrm{H} 5), 1.18(\mathrm{~d}, J=7.2 \mathrm{~Hz}, 3 \mathrm{H}, \mathrm{H} 8) ;{ }^{13} \mathrm{C}$ NMR $\left(75 \mathrm{MHz}, \mathrm{CDCl}_{3}\right) \delta 179.1$ (C), $165.9(\mathrm{C}), 149.3(\mathrm{CH}), 130.1(\mathrm{C}), 52.5\left(\mathrm{CH}_{3}\right), 43.0(\mathrm{CH}), 33.5\left(\mathrm{CH}_{2}\right), 32.5\left(\mathrm{CH}_{2}\right), 31.3(\mathrm{CH})$, $22.1\left(\mathrm{CH}_{3}\right) ; \mathrm{MS}(\mathrm{ESI}+) \mathrm{m} / \mathrm{z} 231\left([\mathrm{M}+\mathrm{H}]^{+}, 100 \%\right)$.

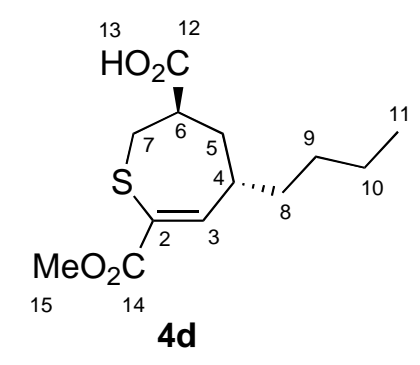

Thiepine 4d: ${ }^{1} \mathrm{H}$ NMR (300 MHz, $\left.\mathrm{CDCl}_{3}\right) \delta 11.1$ (broad s, $1 \mathrm{H}, \mathrm{H} 13$ ), 7.00 (d, $J=5.1 \mathrm{~Hz}, 1 \mathrm{H}$, H3), 3.73 (s, 3H, H15), 3.22 (dd, $J=13.9,7.4$ Hz, 1H, H7), 2.99-3.10 (m, 1H, H6), 2.84 (dd, $J=$ 13.9, 3.8 Hz, 1H, H7), 2.69-2.83 (m, 1H, H4), 2.06 (ddd, $J=14.2$, 5.1, 1.8 Hz, 1H, H5), 1.89 (ddd, $J=14.2,10.5,5.4$ Hz, 1H, H5), 1.29-1.62 (m, 2H, H8), 1.19-1.36 (m, 4H, H9-H10), 0.800.91 (m, 3H, H11); ${ }^{13} \mathrm{C}$ NMR (75 MHz, $\mathrm{CDCl}_{3}$ ) $\delta 179.5$ (C), 165.9 (C), 149.0 (CH), 130.4 (C), $52.5\left(\mathrm{CH}_{3}\right), 43.0(\mathrm{CH}), 36.4(\mathrm{CH}), 35.9\left(\mathrm{CH}_{2}\right), 33.3\left(\mathrm{CH}_{2}\right), 30.8\left(\mathrm{CH}_{2}\right), 28.9\left(\mathrm{CH}_{2}\right), 22.6\left(\mathrm{CH}_{2}\right)$, 
$13.8\left(\mathrm{CH}_{3}\right)$; MS (ESI+) m/z $273\left([\mathrm{M}+\mathrm{H}]^{+}, 100 \%\right)$.

Structure revision of the cycloheptenic acid $4\left(\mathrm{X}=\mathrm{Y}=\mathrm{CH}_{2} ; \mathrm{R}^{2}=\mathrm{CH}_{3}\right)$ :

The initially reported ${ }^{18 \mathrm{~b}}$ incorrect cis stereochemistry of the carbocyclic analog 4 ( $\mathrm{X}=\mathrm{Y}=\mathrm{CH}_{2}$; $\mathrm{R}^{2}=\mathrm{CH}_{3}$ ) has been revisited and confirmed to be trans by X-ray diffraction analysis of the diol A obtained by dihydroxylation of the cycloheptenic acid $\mathbf{4}\left(\mathrm{X}=\mathrm{Y}=\mathrm{CH}_{2} ; \mathrm{R}^{2}=\mathrm{CH}_{3}\right)$.
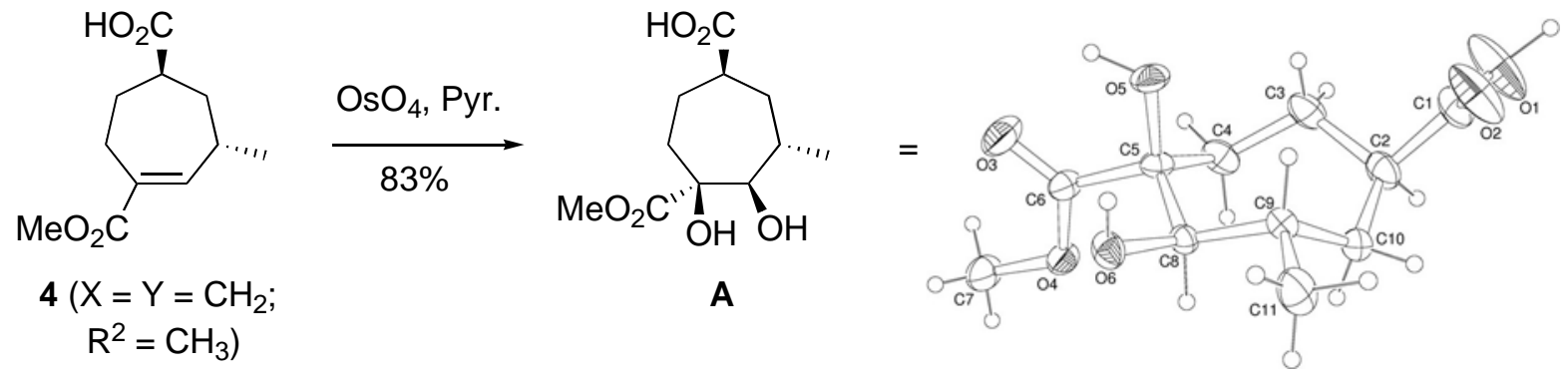

Diol A: needles from AcOEt:hexane by slow evaporation at room temperature; mp $137{ }^{\circ} \mathrm{C} ;{ }^{1} \mathrm{H}$ NMR (300 MHz, $\mathrm{CDCl}_{3}$ ) $\delta 3.81$ (s, 3H), 3.49 (broad s, 1H), 3.44 (d, $J=9.9 \mathrm{~Hz}, 1 \mathrm{H}$ ), 2.67 (dddd, $J=10.5,6.7,6.7,3.1 \mathrm{~Hz}, 1 \mathrm{H}), 2.20$ (dddd, $J=14.3,10.9,10.8,2.7 \mathrm{~Hz}, 1 \mathrm{H}), 1.70-2.05(\mathrm{~m}, 6 \mathrm{H})$, 1.59 (ddd, $J=15.6,9.1,6.8 \mathrm{~Hz}, 1 \mathrm{H}), 1.06$ (d, $J=6.9 \mathrm{~Hz}, 3 \mathrm{H}), \mathrm{CO}_{2} \mathrm{H}$ masked; ${ }^{13} \mathrm{C}$ NMR $(75$ MHz, $\left.\left(\mathrm{CD}_{3}\right)_{2} \mathrm{CO}\right) \delta 20.5\left(\mathrm{CH}_{3}\right), 22.9\left(\mathrm{CH}_{2}\right), 32.7(\mathrm{CH}), 33.2\left(\mathrm{CH}_{2}\right), 35.1\left(\mathrm{CH}_{2}\right), 41.4(\mathrm{CH}), 52.6$ ( $\left.\mathrm{CH}_{3}\right), 79.9$ (C), 81.2 (CH), 177.3 (C), 177.5 (C); MS (ESI-) m/z 245 ([M-H]', 100\%).

The ellipsoids in the ORTEP view of $\mathbf{A}$ are at 30\% probability. The CIF for $\mathbf{A}$ is available free of charge via the Internet at http://pubs.acs.org or http://www.ccdc.cam.ac.uk under the CCDC number 615710. 

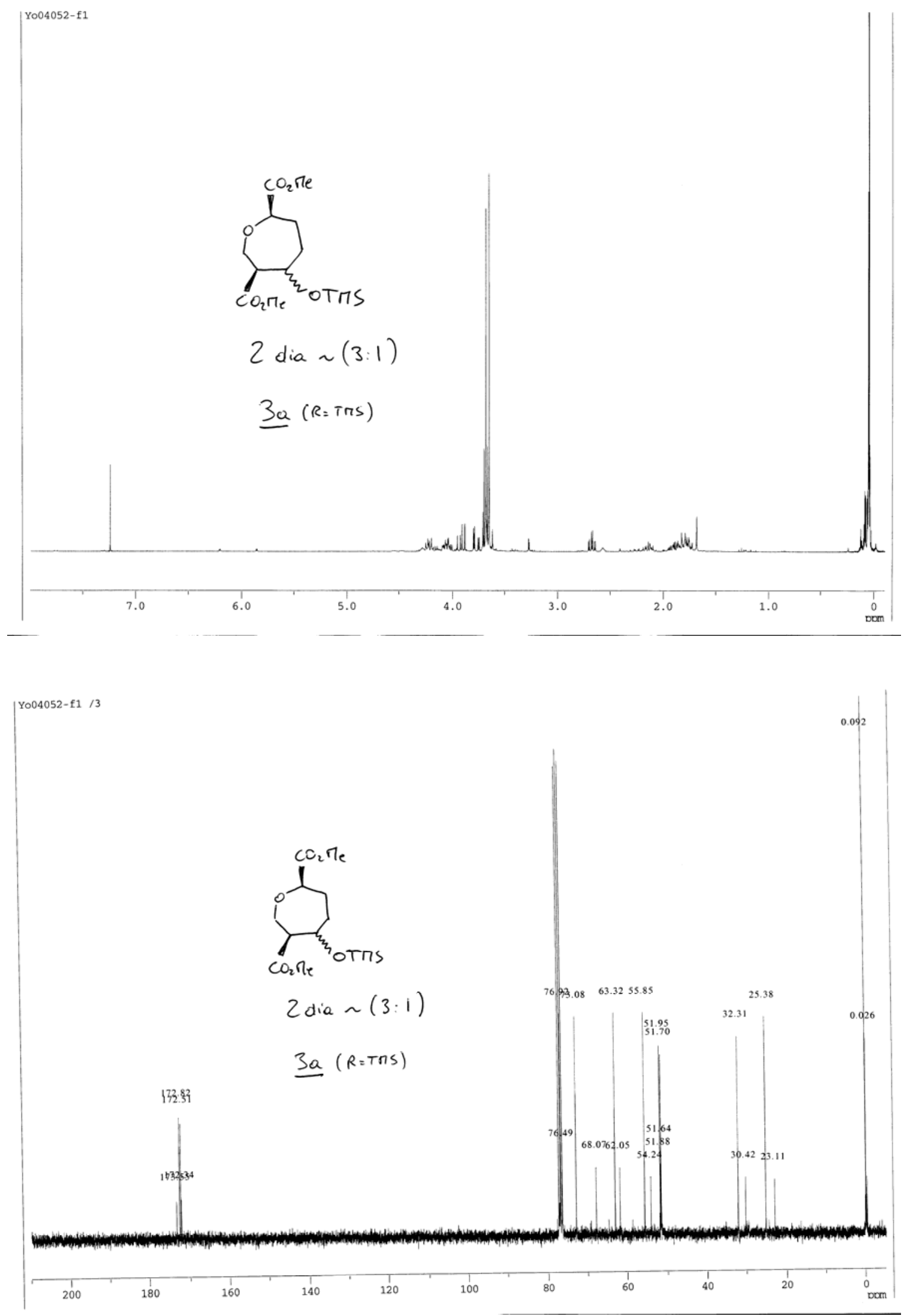

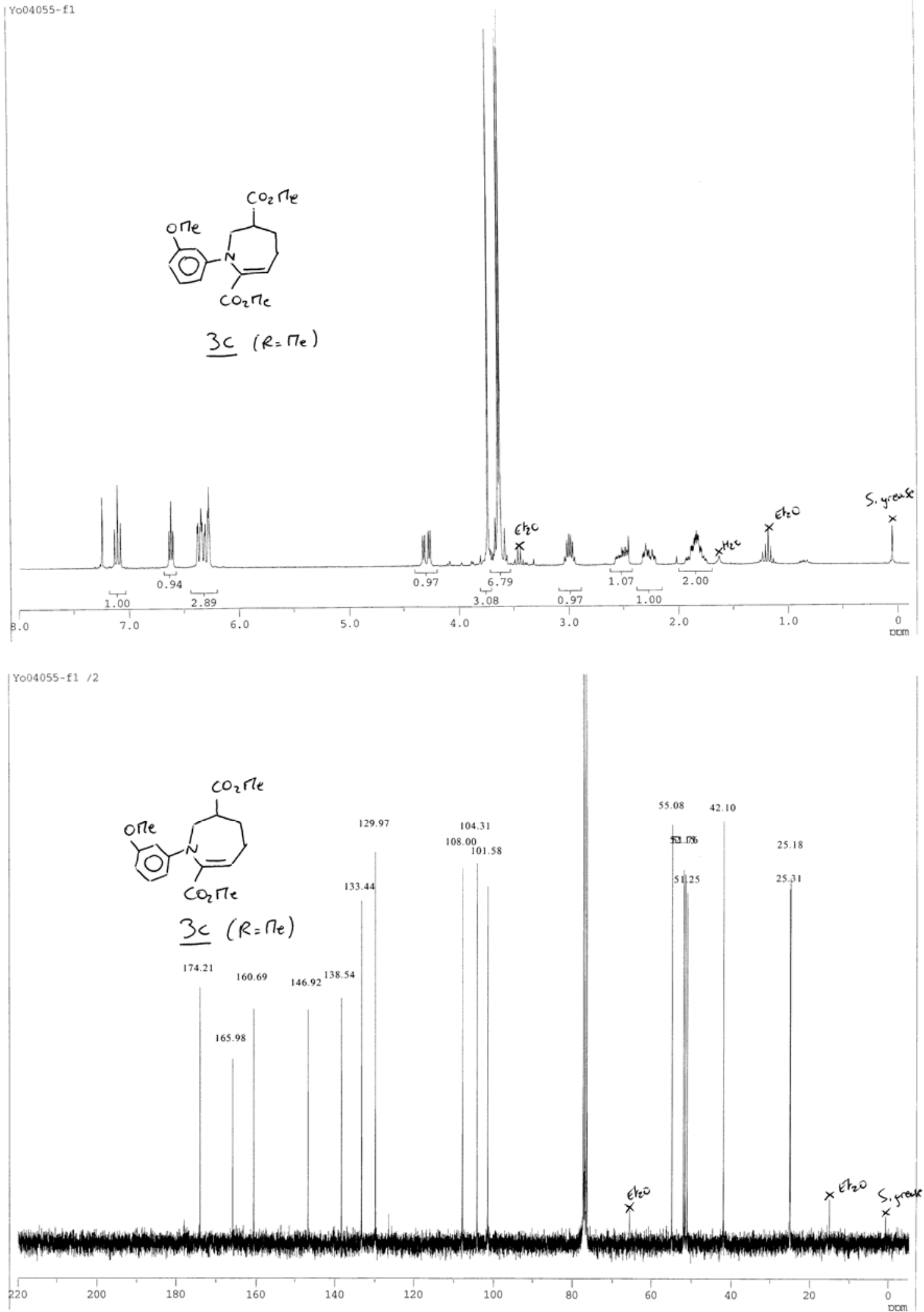

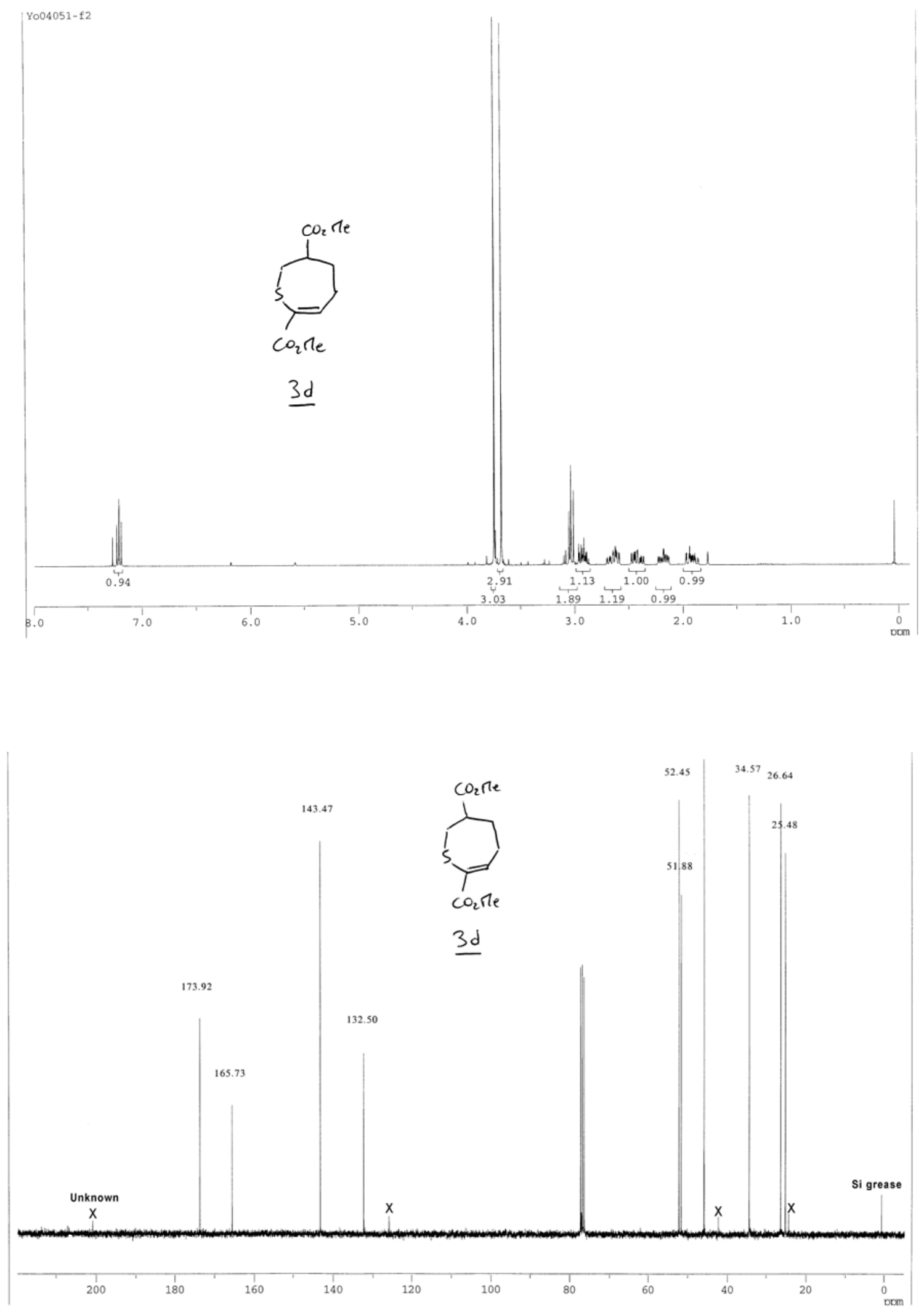

- S16 - 

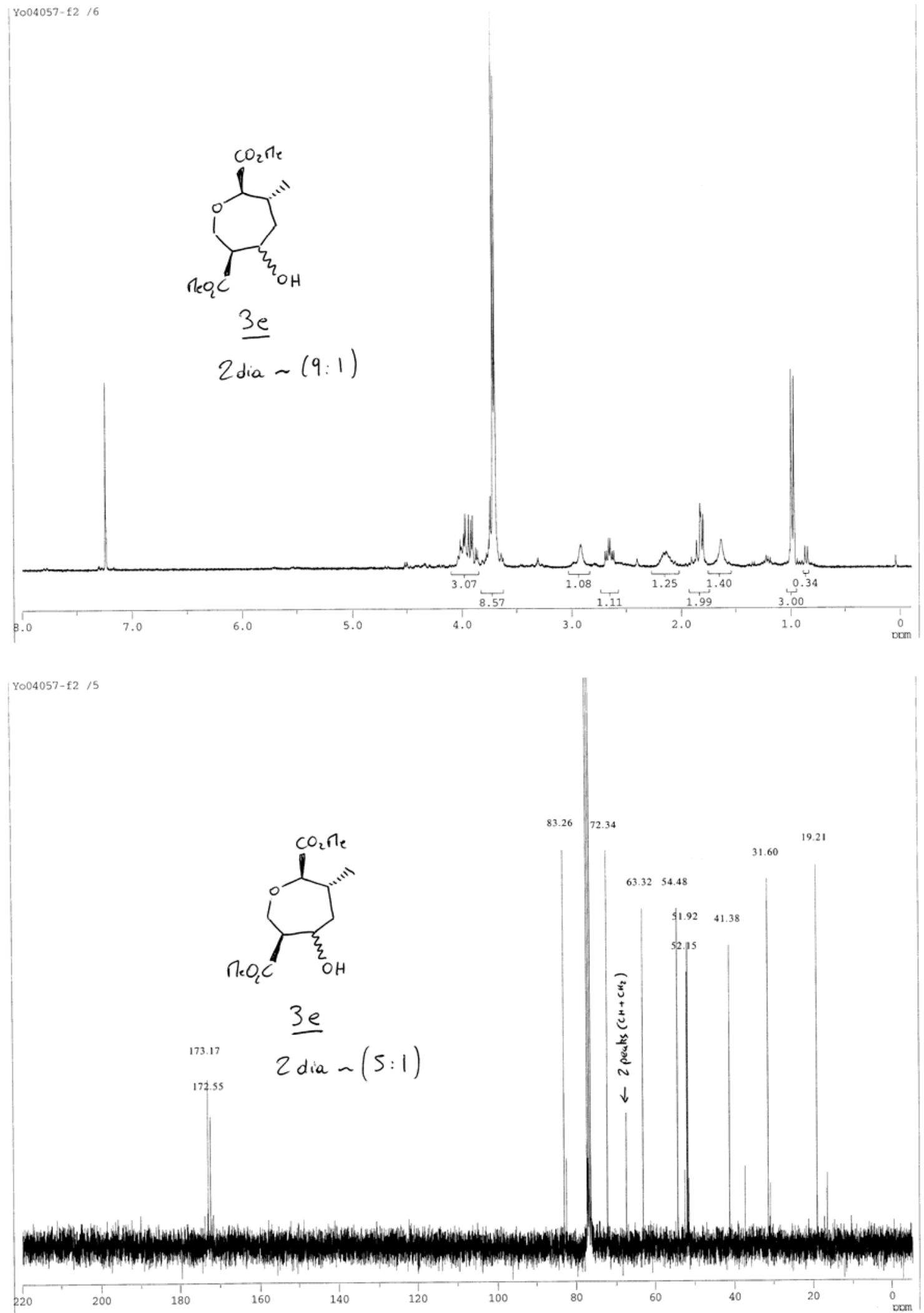

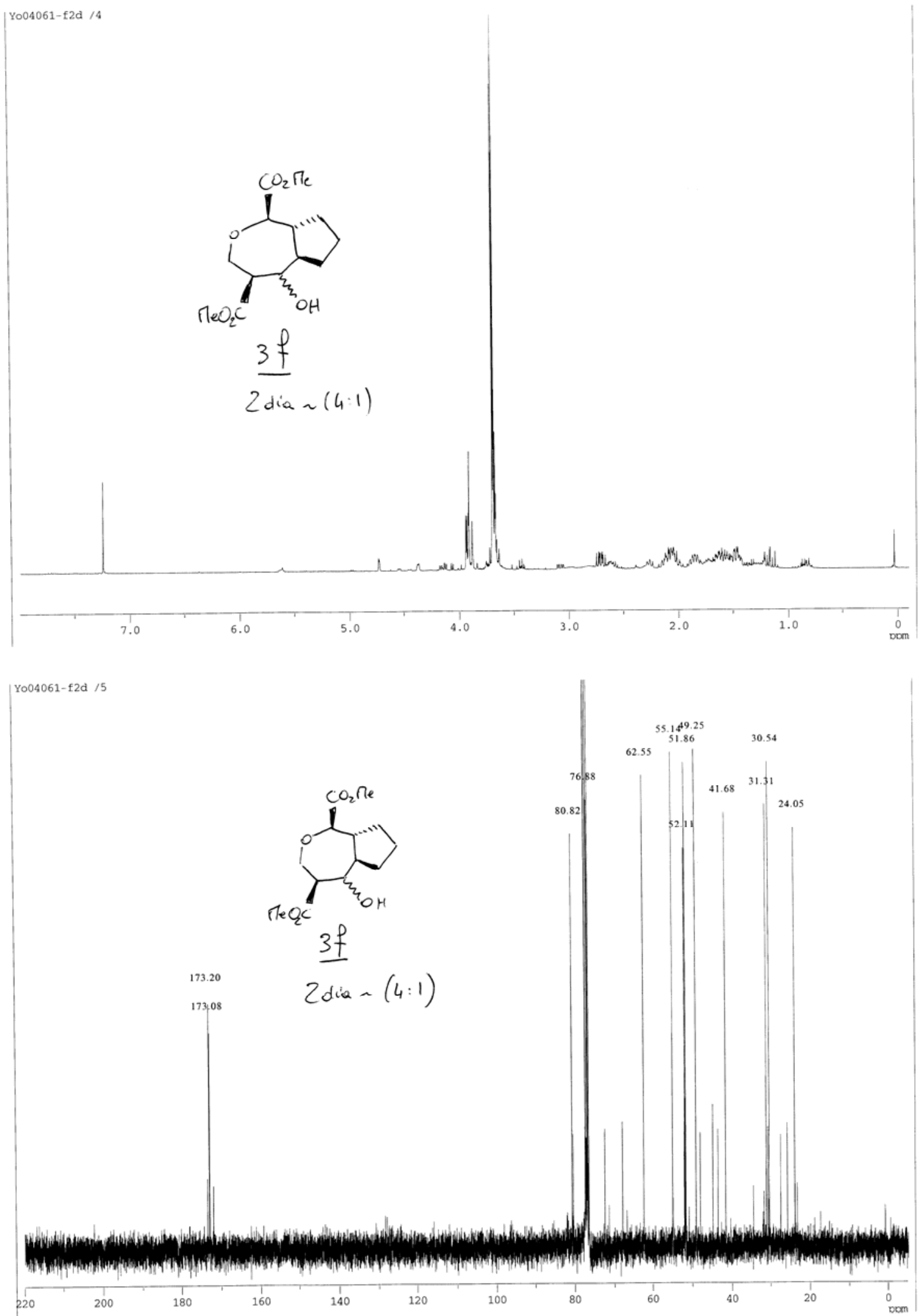

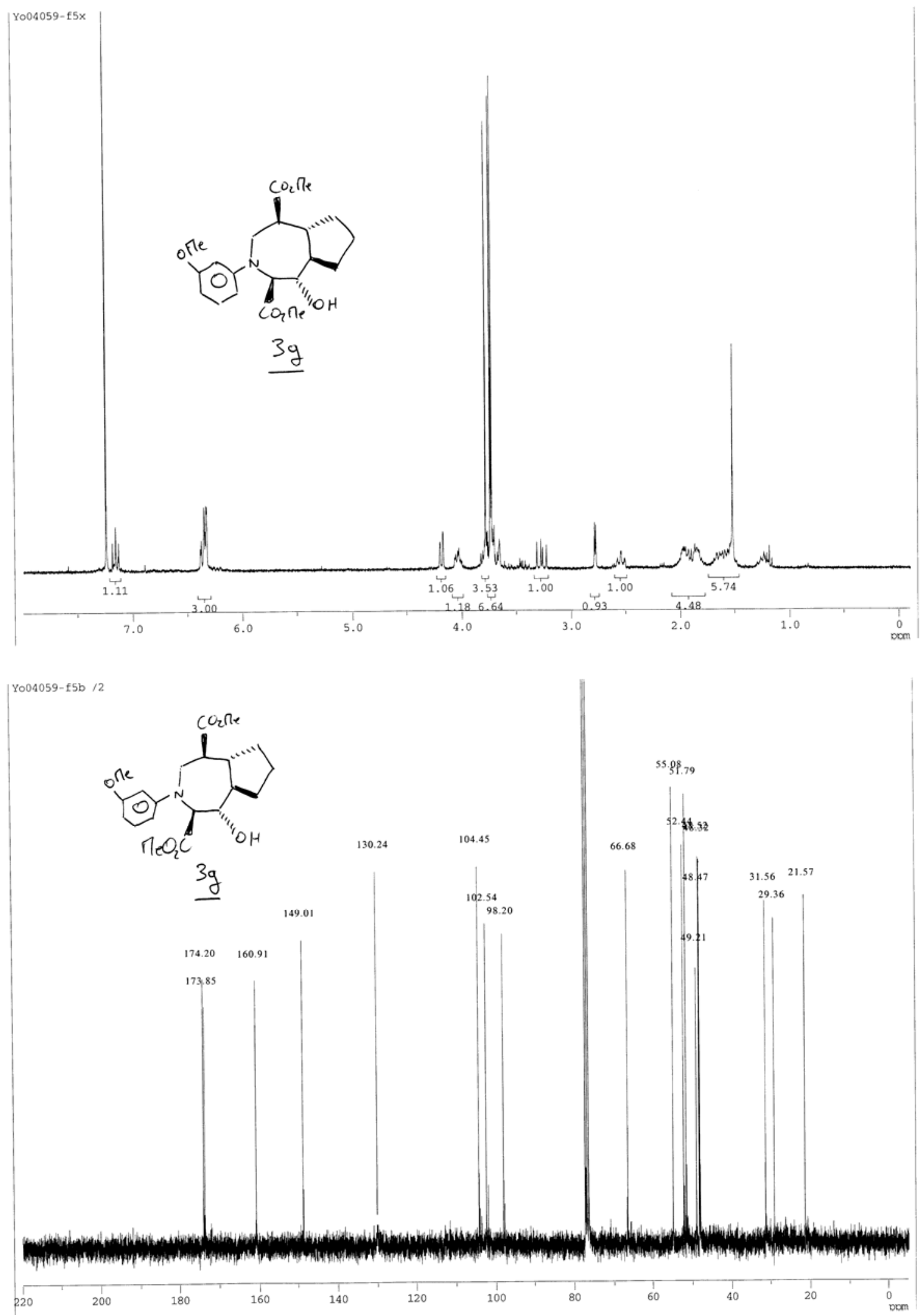

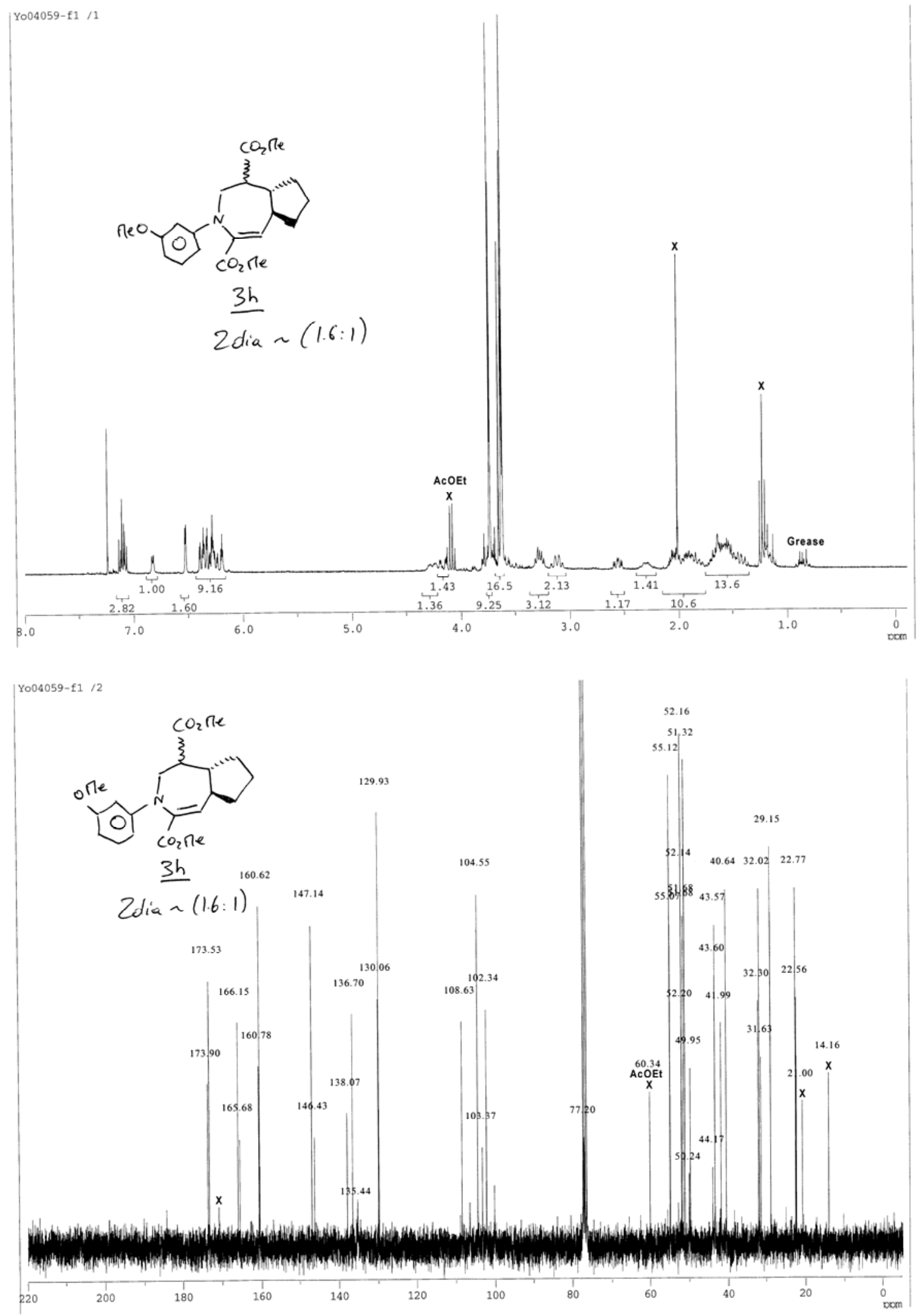

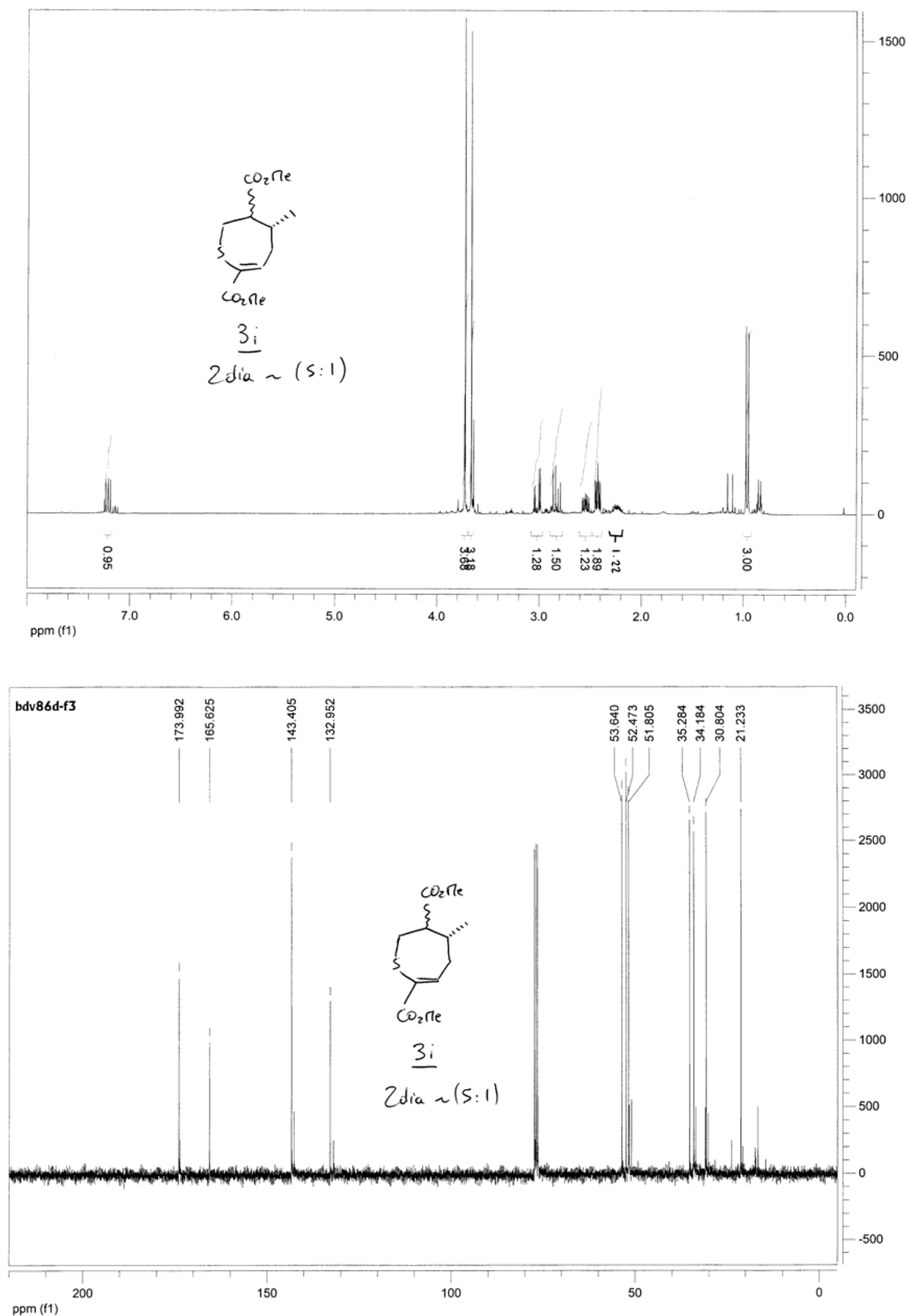

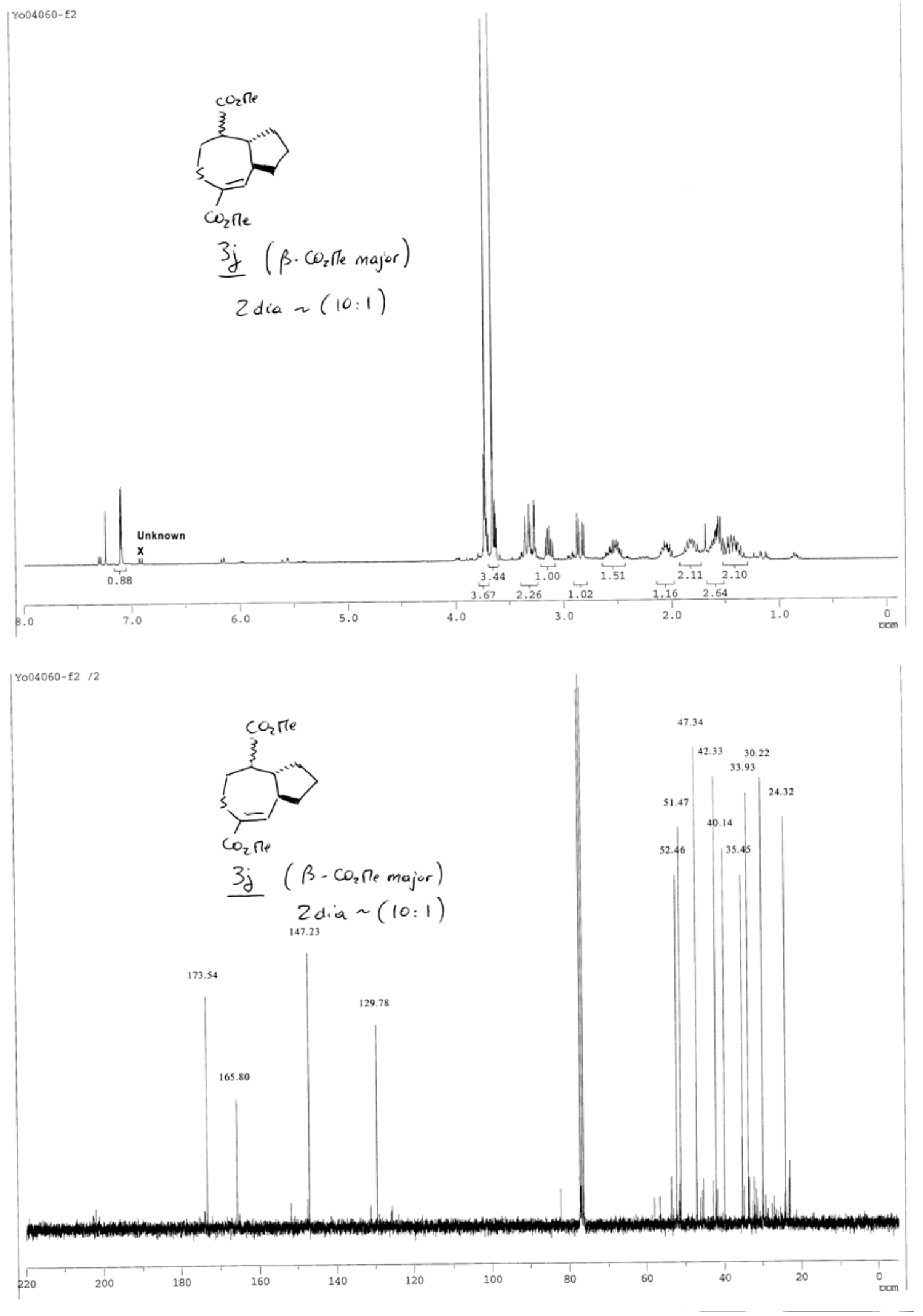

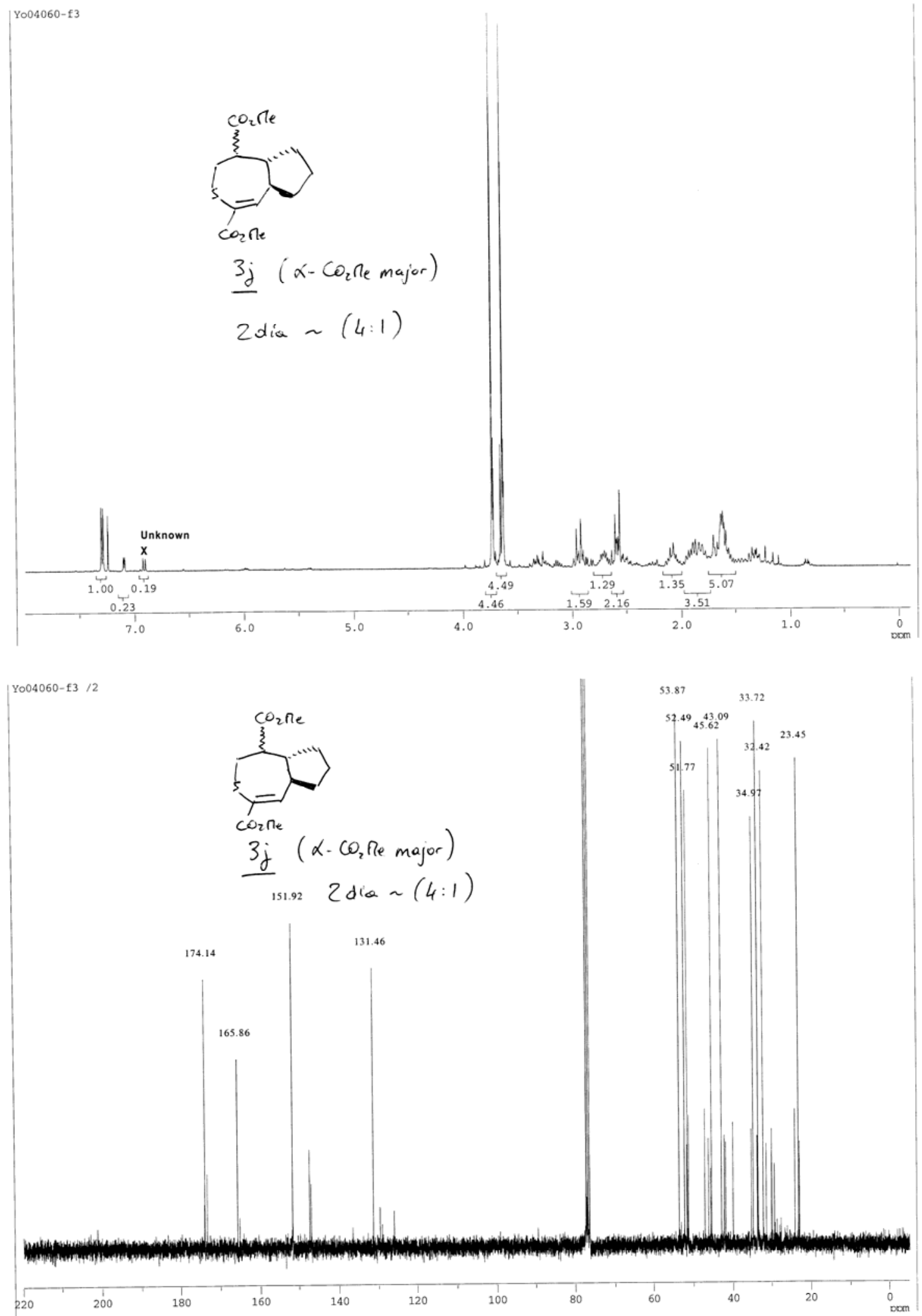

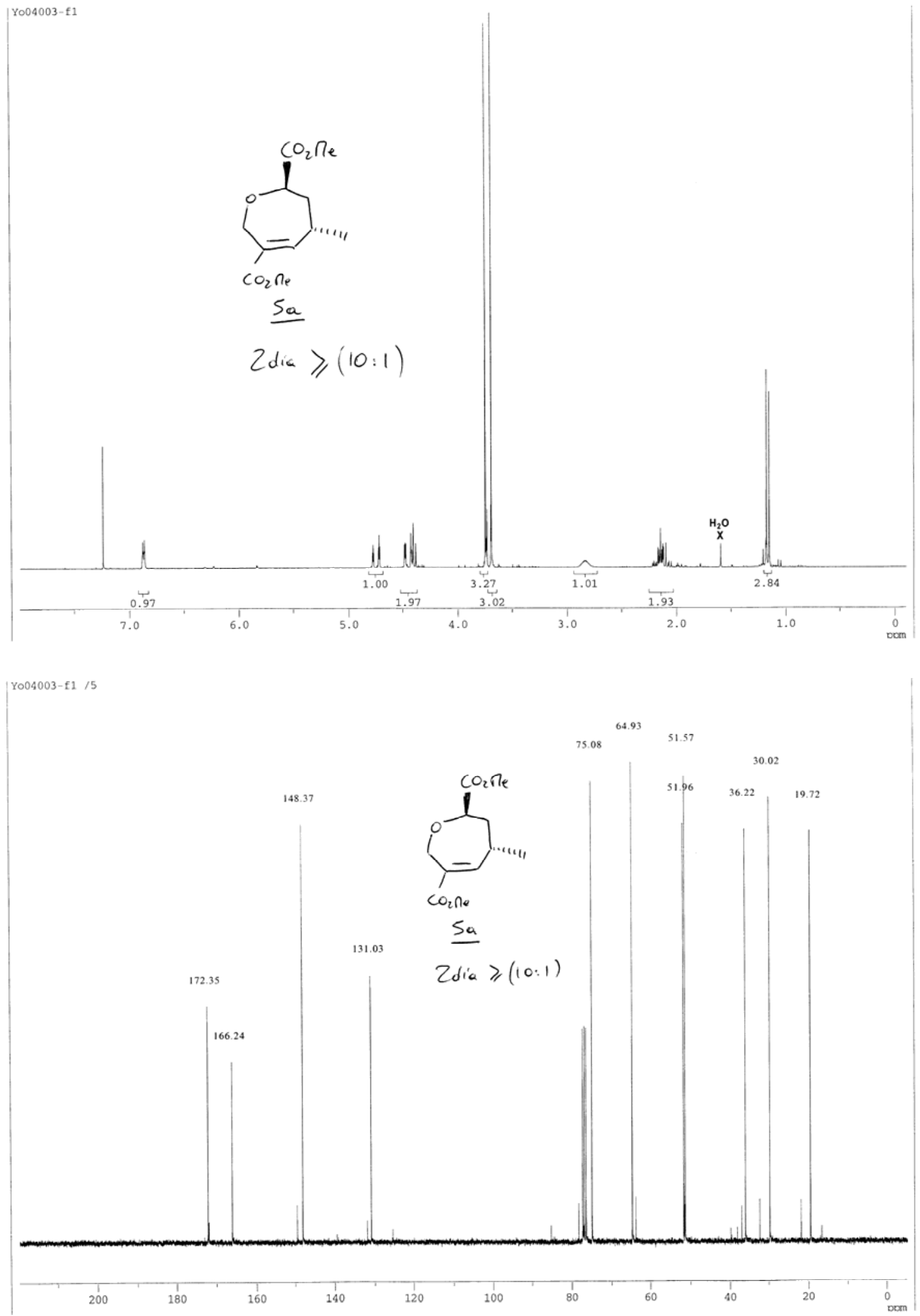

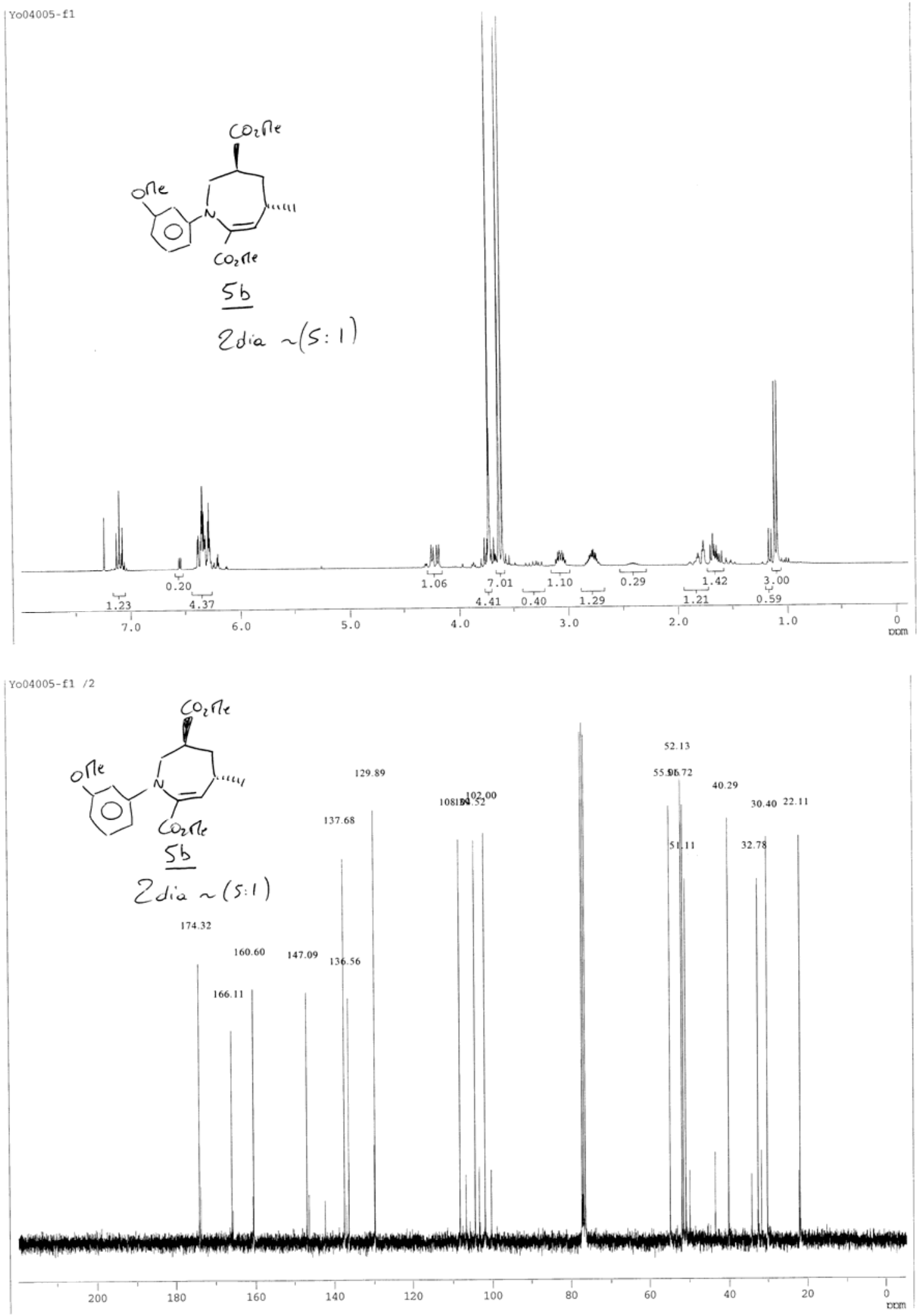

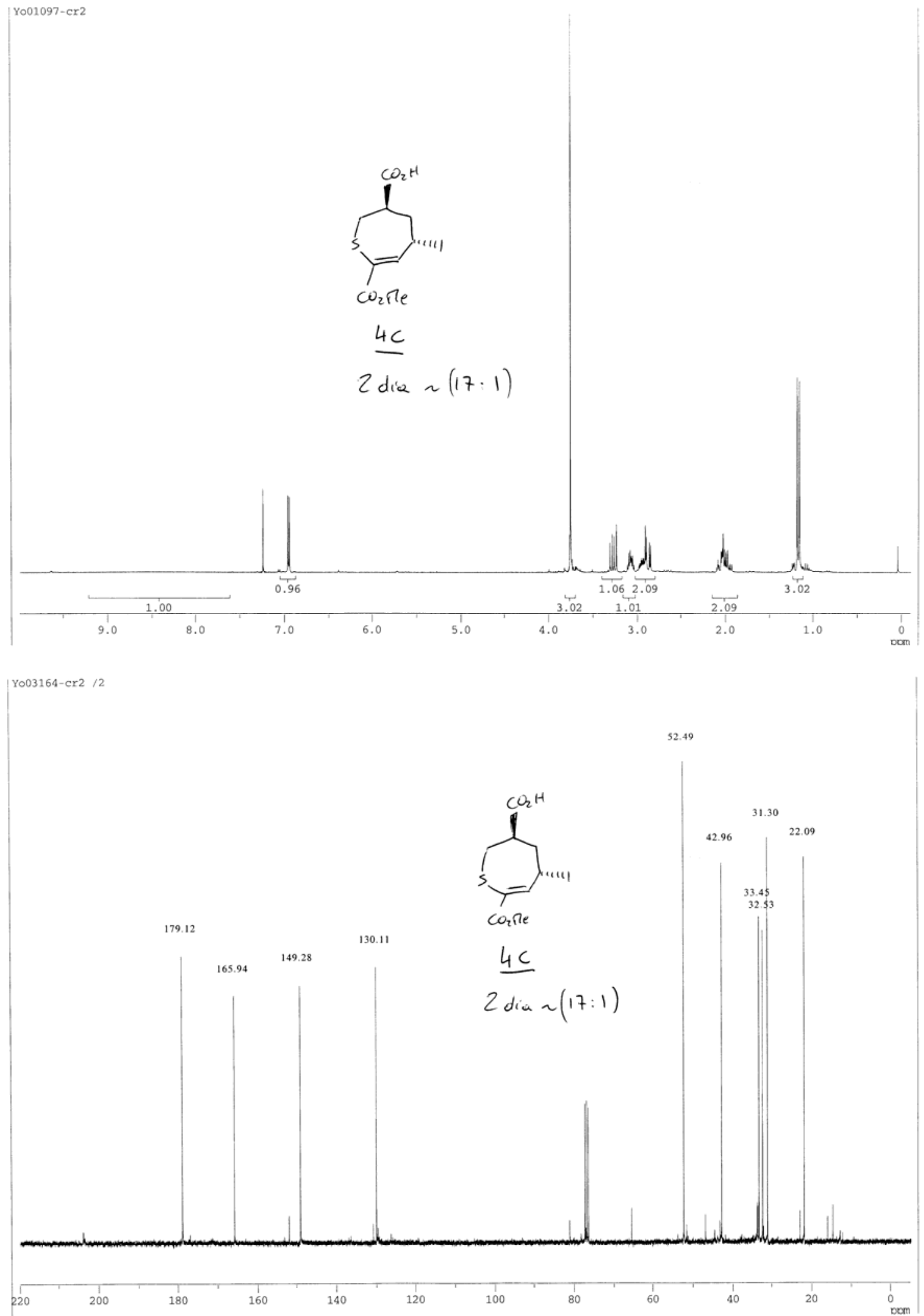

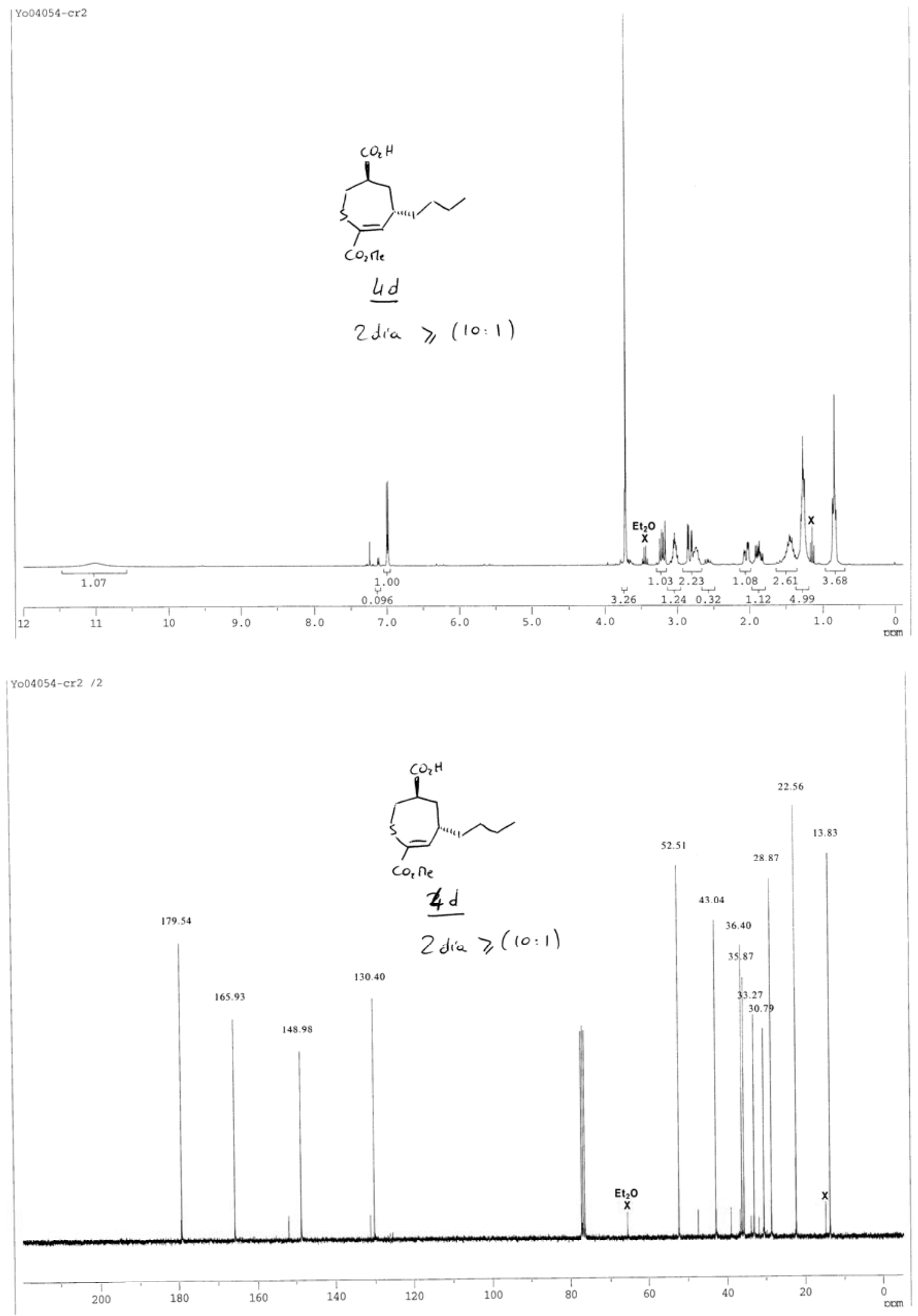

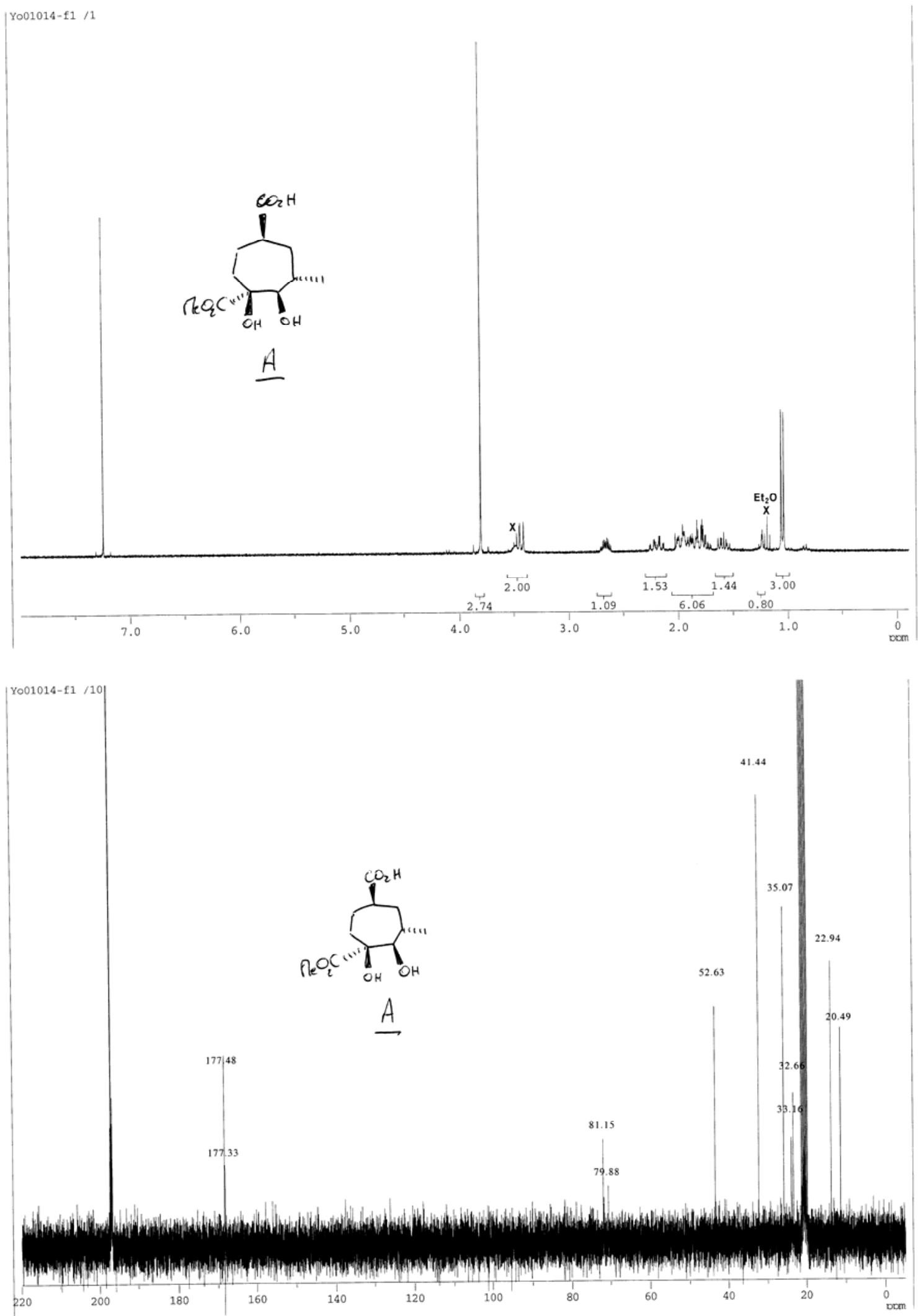
NOESY spectrum of $3 e(500 \mathrm{MHz}$ in $\mathrm{CDCl} 3-\mathrm{C} 6 \mathrm{D} 6)$

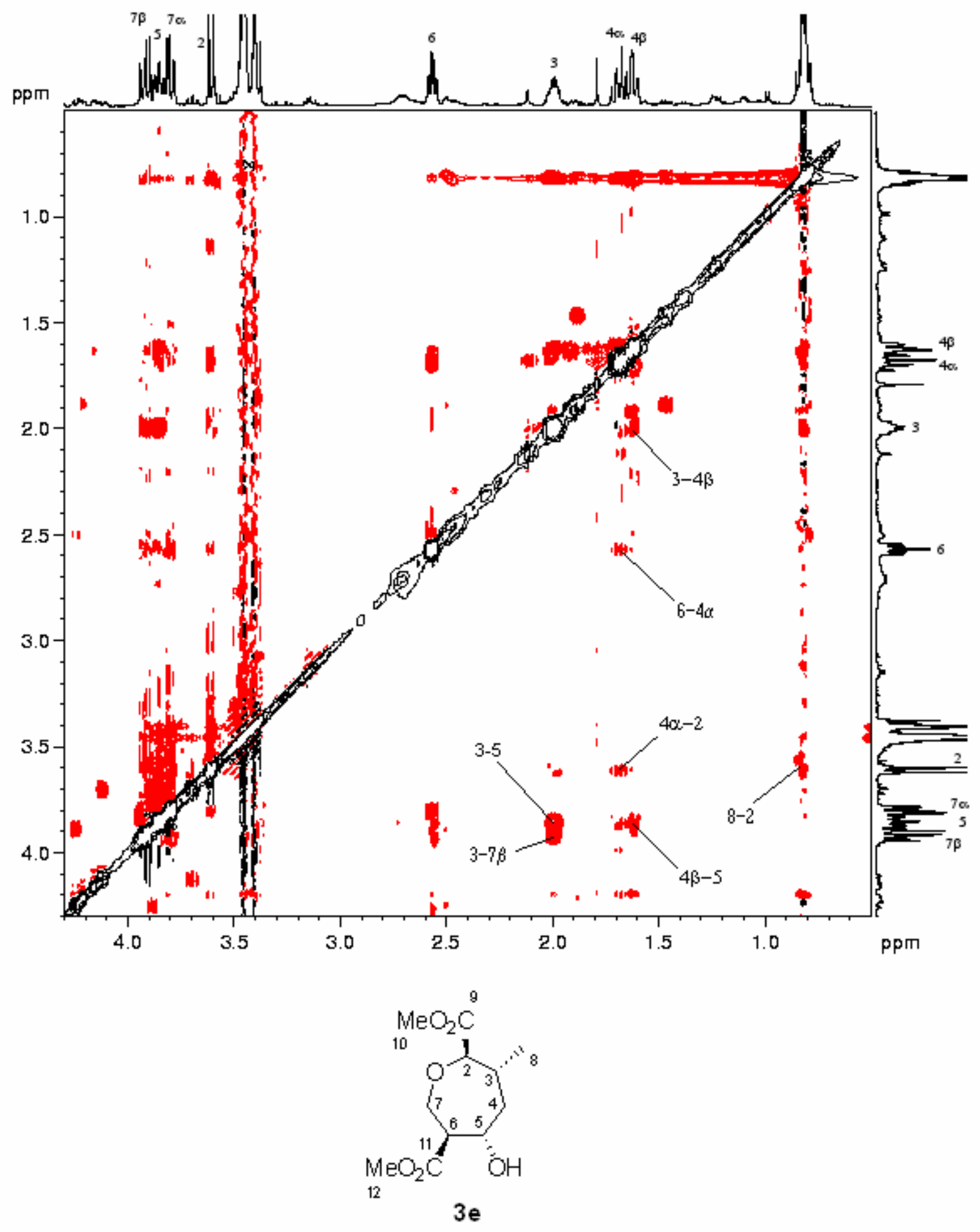


NOESY spectrum of $3 \mathrm{f}(500 \mathrm{MHz}$ in $\mathrm{CDCl} 3)$

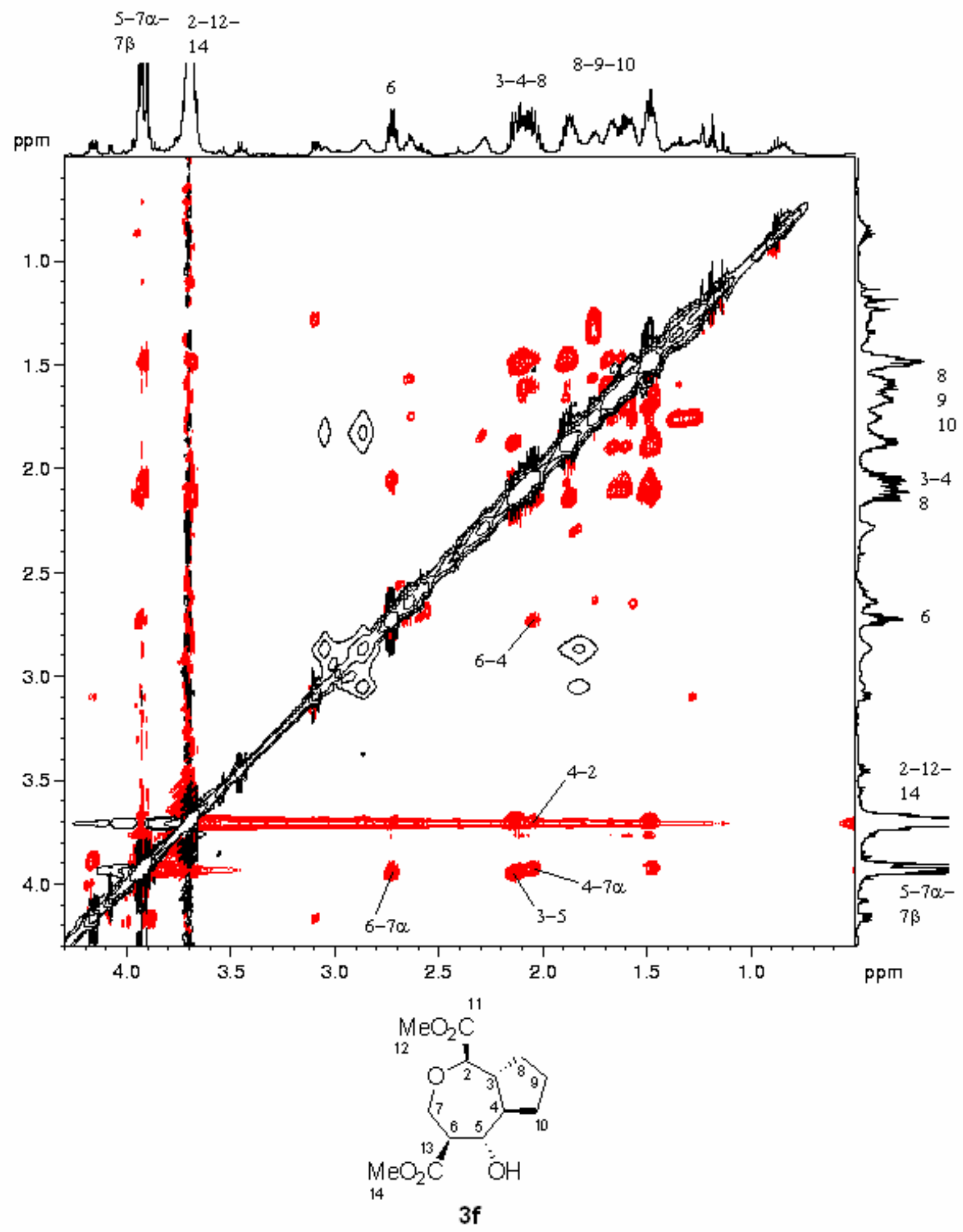


NOESY spectrum of $3 \mathrm{~h}(500 \mathrm{MHz}$ in $\mathrm{CDCl} 3)$

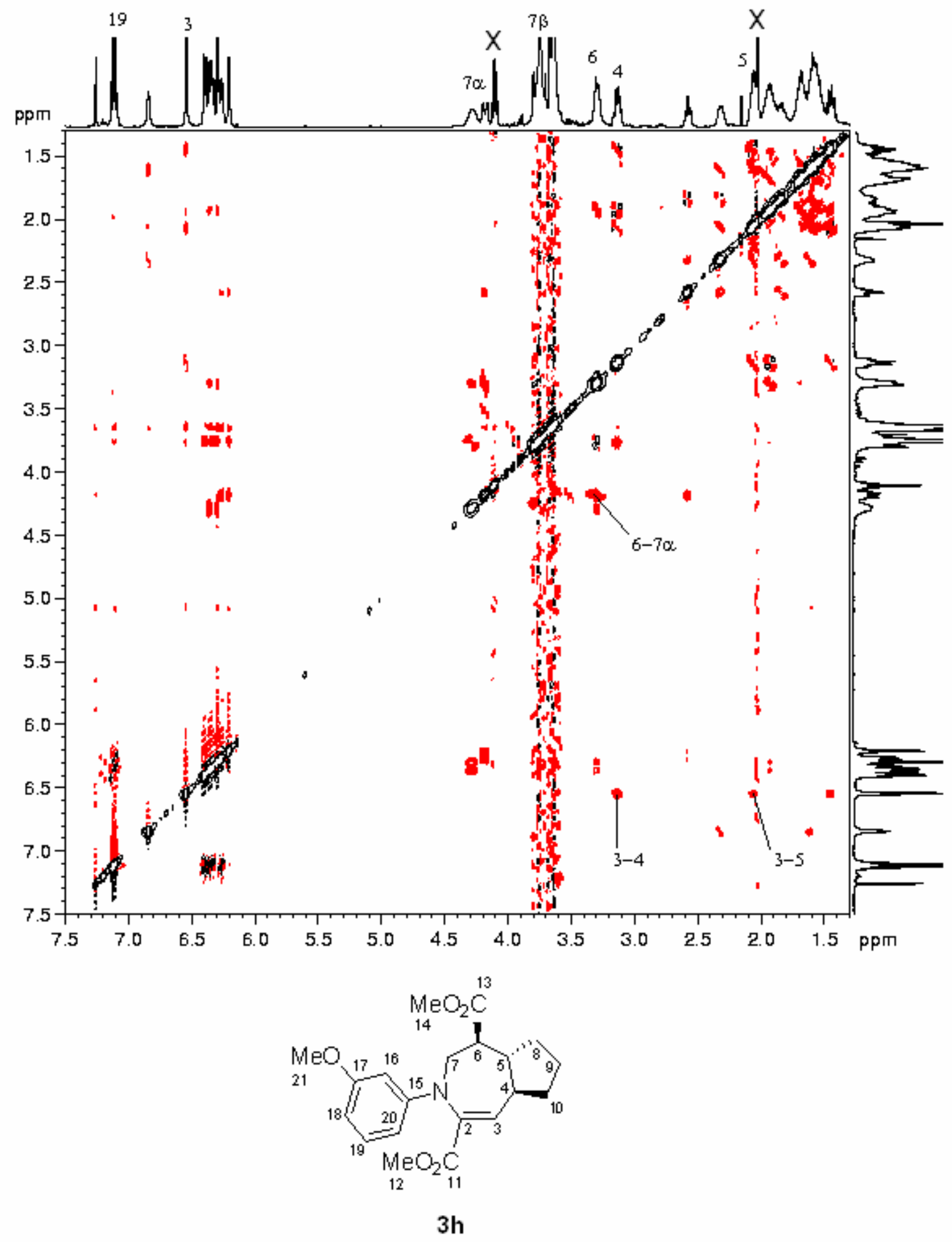


NOESY spectrum of $3 \mathbf{j}$ (beta-CO2Me, $500 \mathrm{MHz}$ in $\mathrm{CDCl} 3$ )

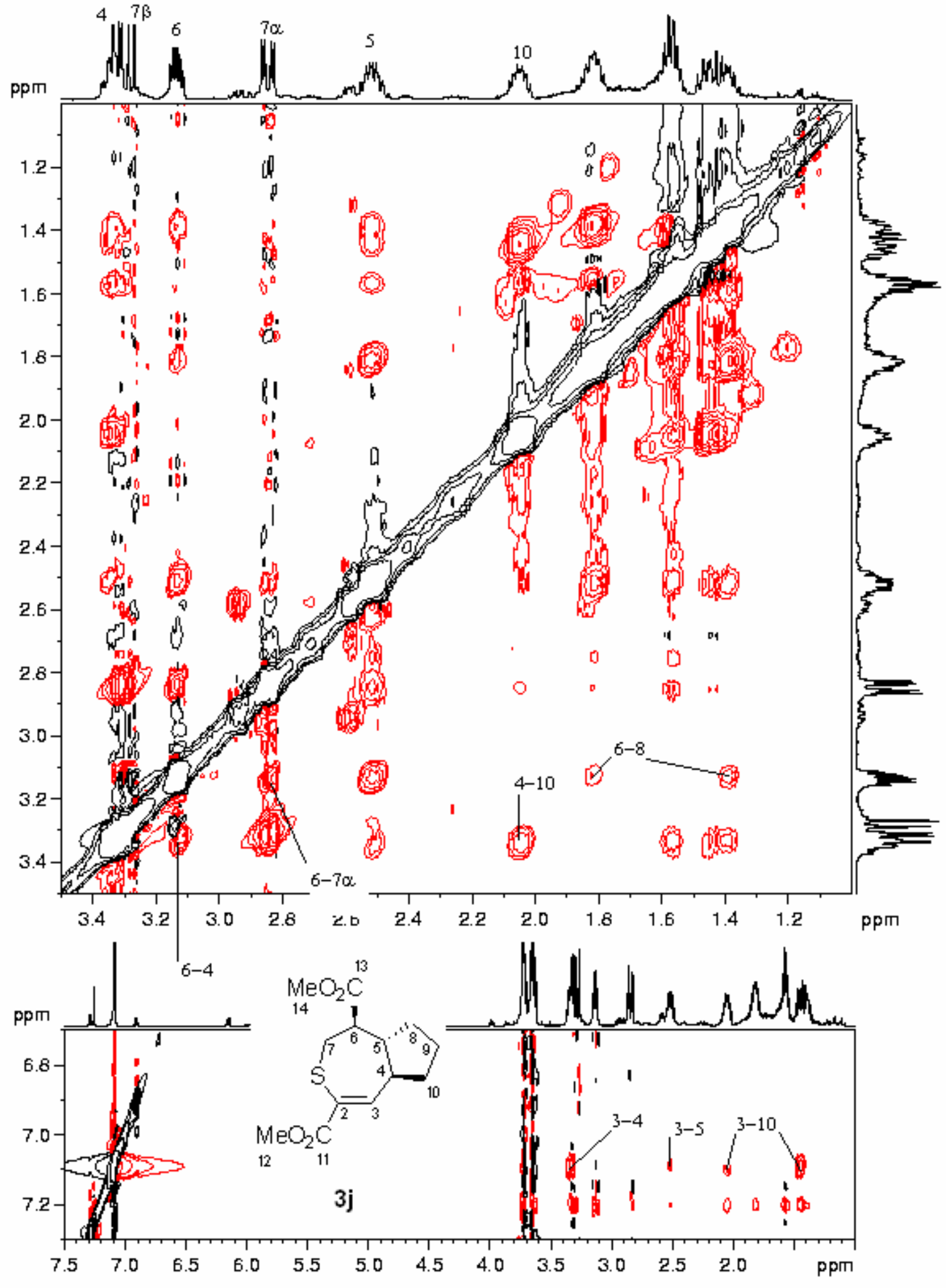


NOESy spectrum of $3 \mathrm{j}$ (alpha-CO2Me, $500 \mathrm{MHz}$ in $\mathrm{CDCl}$ )
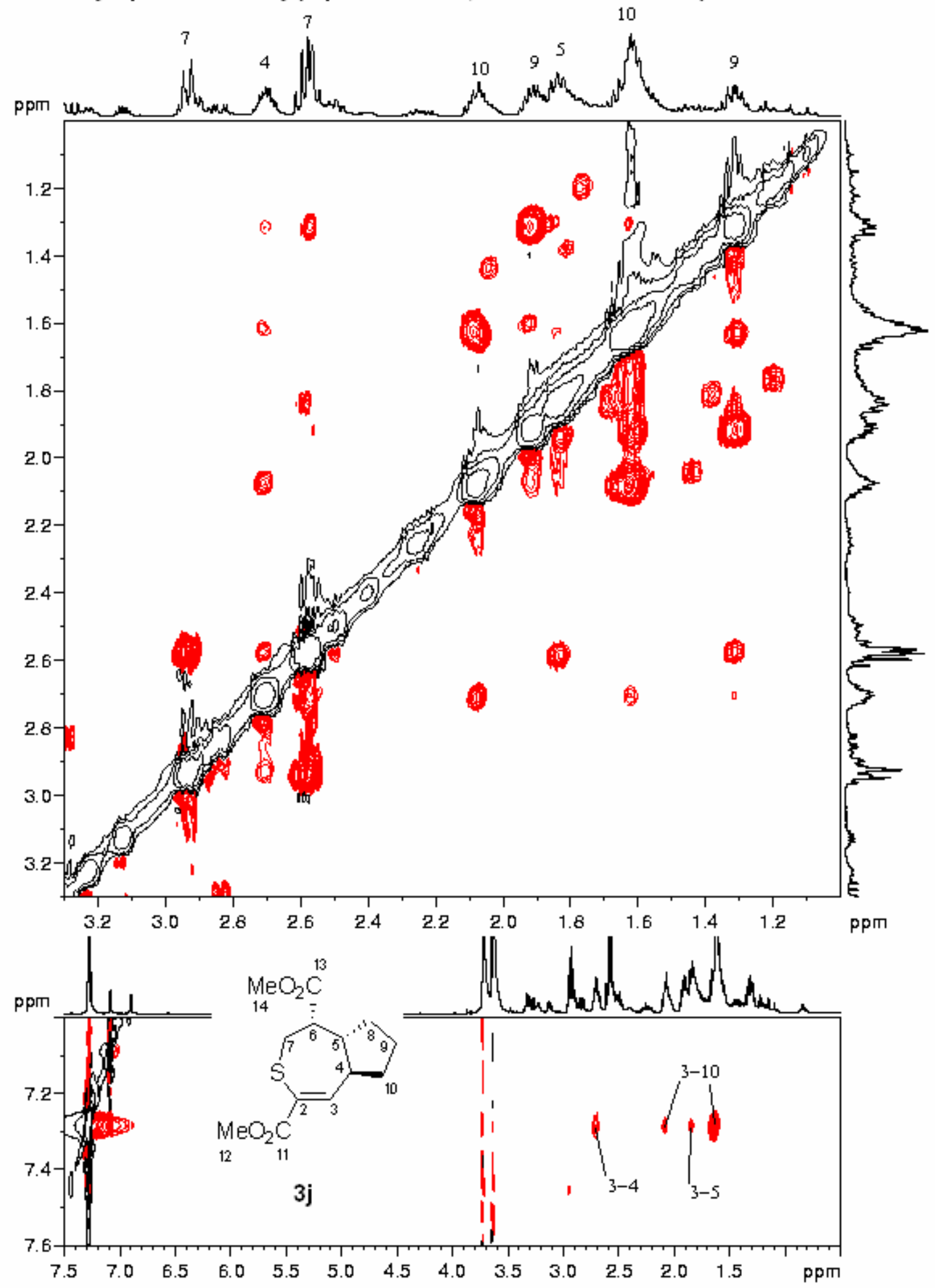
NOESY spectrum of $5 \mathrm{a}(300 \mathrm{MHz}, \mathrm{CDCl})$
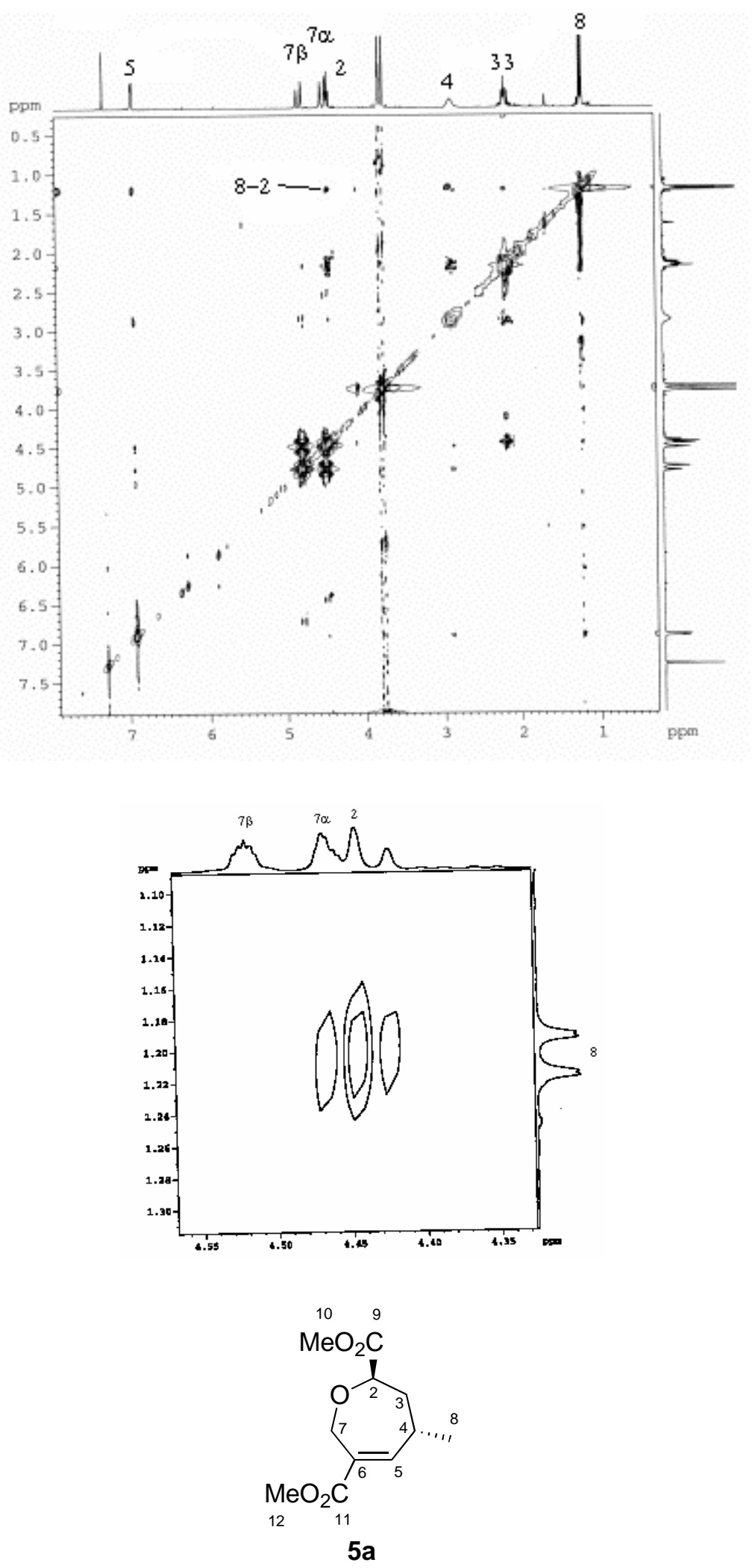
NOESY spectrum of $5 b(300 \mathrm{MHz}, \mathrm{CDCl} 3)$

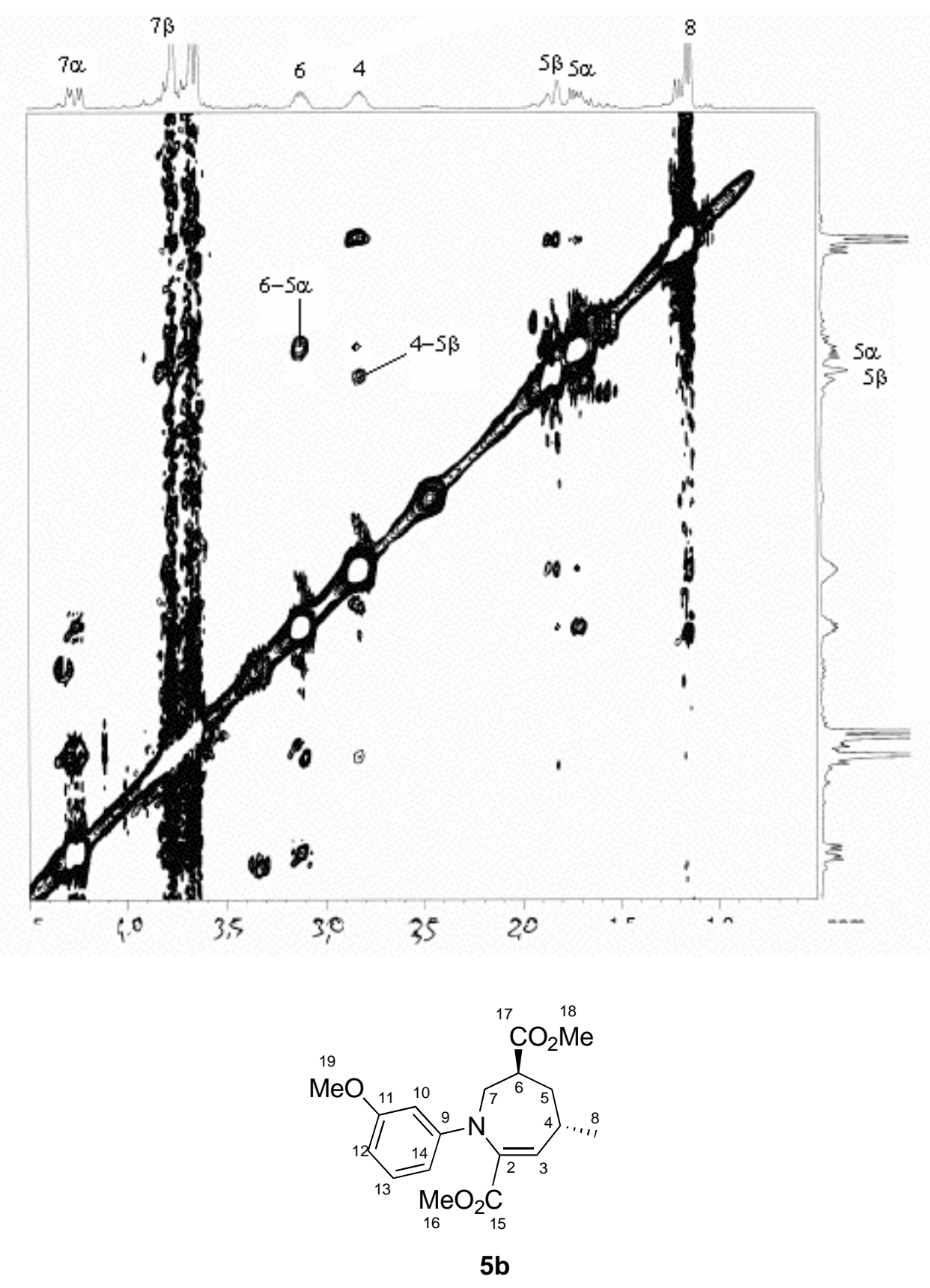




\section{NOESY spectrum of $4 \mathrm{c}(500 \mathrm{MHz}, \mathrm{CDCl} 3)$}

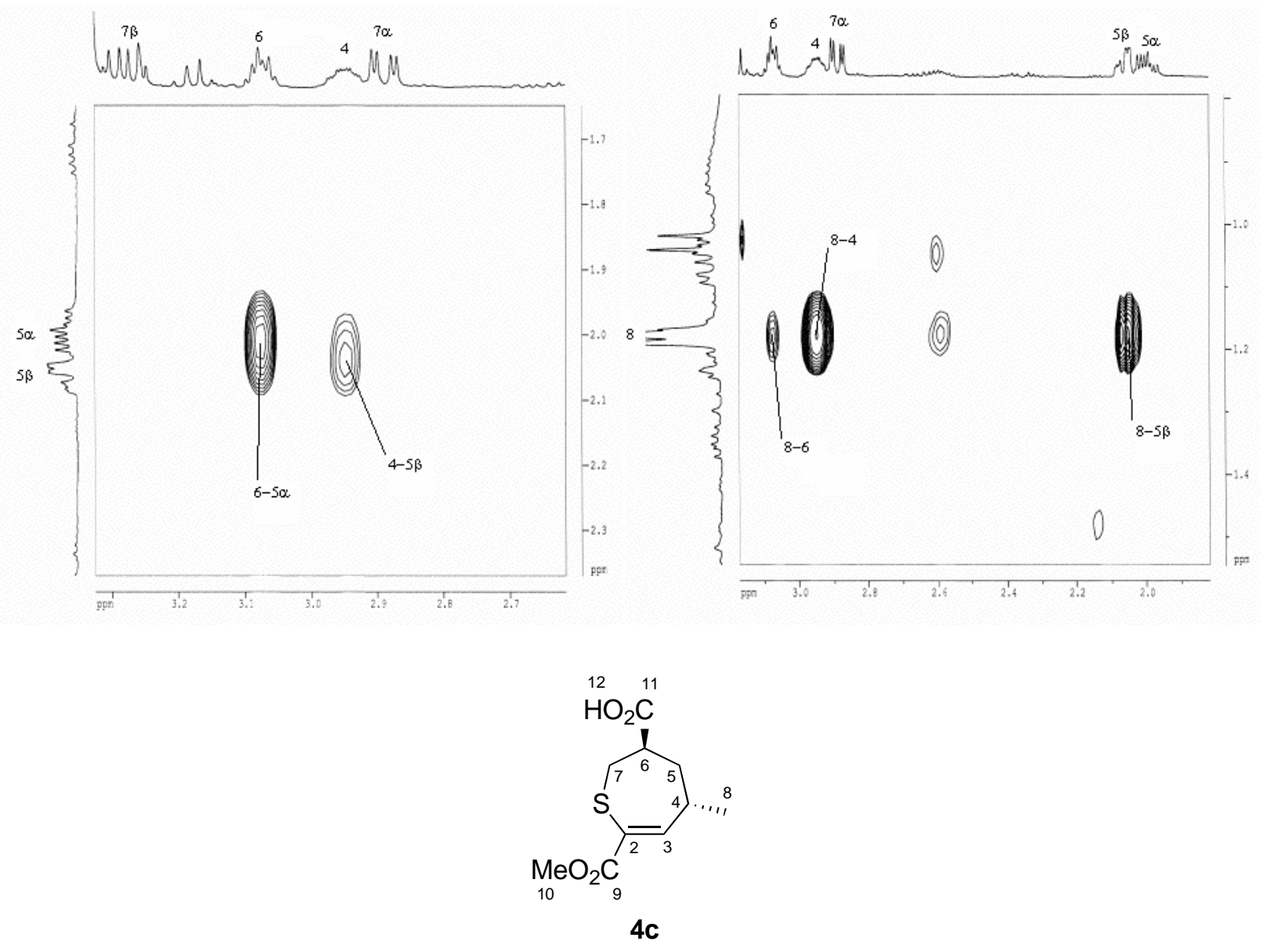




\section{NOESY spectrum of $4 \mathrm{~d}(300 \mathrm{MHz}, \mathrm{CDCl} 3)$}
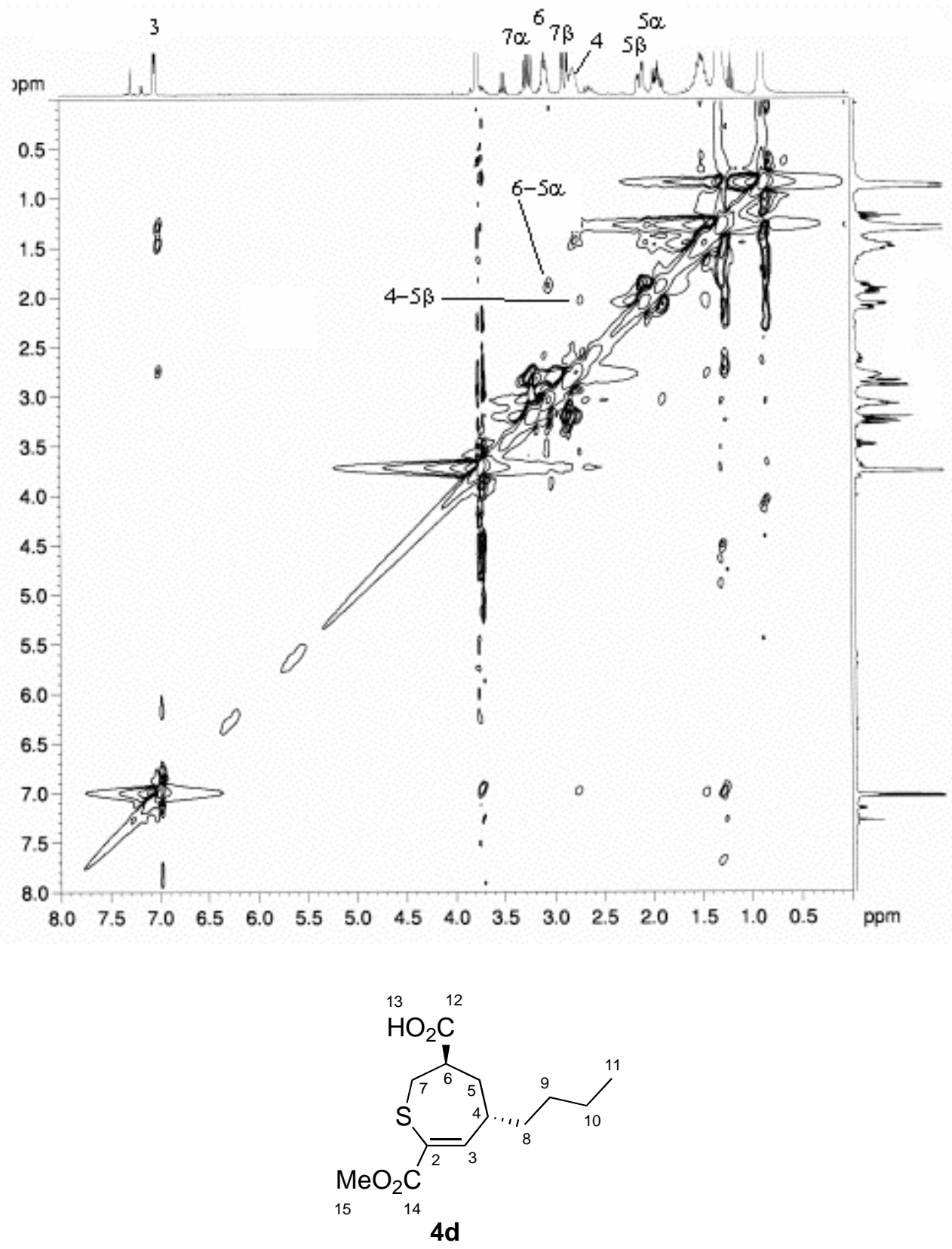